\title{
ON THE DOUBLE TRANSFER AND THE $f$-INVARIANT
}

\author{
GEOFFREY POWELL \\ Laboratoire Analyse, Géométrie et Applications, UMR 7539, Institut Galilée, \\ Université Paris 13, 93430 Villetaneuse, France \\ e-mail:powell@math.univ-paris13.fr
}

(Received 5 May 2011; accepted 24 September 2011; first published online 30 March 2012)

\begin{abstract}
The purpose of this paper is to investigate the algebraic double $S^{1}$ transfer, in particular the classes in the two-line of the Adams-Novikov spectral sequence which are the image of comodule primitives of the $M U$-homology of $\mathbb{C} \mathbf{P}^{\infty} \times \mathbb{C} \mathbf{P}^{\infty}$ via the algebraic double transfer. These classes are analysed by two related approaches: the first, $p$-locally for $p \geq 3$, by using the morphism induced in $M U$ homology by the chromatic factorisation of the double transfer map together with the $f^{\prime}$-invariant of Behrens (for $p \geq 5$ ) (M. Behrens, Congruences between modular forms given by the divided $\beta$-family in homotopy theory, Geom. Topol. 13(1) (2009), 319357). The second approach (after inverting 6) uses the algebraic double transfer and the $f$-invariant of Laures (G. Laures, The topological q-expansion principle, Topology 38(2) (1999), 387-425).
\end{abstract}

2000 Mathematics Subject Classification. Primary 55R12; Secondary 55N34, $55 \mathrm{P} 42$.

1. Introduction. The double $S^{1}$-transfer is a stable morphism $\operatorname{tr}_{2}: \mathbb{C} \mathbf{P}_{+}^{\infty} \wedge \mathbb{C} \mathbf{P}_{+}^{\infty} \rightarrow$ $S^{-2}$; a fundamental problem is to determine the image of

$$
\left(\operatorname{tr}_{2}\right)_{*}: \pi_{*}^{\mathrm{s}}\left(\mathbb{C} \mathbf{P}_{+}^{\infty} \wedge \mathbb{C} \mathbf{P}_{+}^{\infty}\right) \rightarrow \pi_{*-2}^{\mathrm{s}}
$$

in stable homotopy.

This has an algebraic counterpart with respect to any complex oriented cohomology theory, in particular complex cobordism $M U$. Namely, there is an algebraic double transfer $\left[e_{\tau}\right]^{2}$, which is a class in $\operatorname{Ext}_{M U_{*} M U}^{2}\left(M U_{*}\left(\mathbb{C} \mathbf{P}_{+}^{\infty} \wedge \mathbb{C P}_{+}^{\infty}\right), M U_{*}[-4]\right)$, where [-4] denotes the shift in internal degree and Ext is calculated in the category of comodules over the Hopf algebroid $\left(M U_{*}, M U_{*} M U\right)$; this induces a morphism

$$
\operatorname{Hom}_{M U_{*} M U}^{*}\left(M U_{*}, M U_{*}\left(\mathbb{C P}_{+}^{\infty} \wedge \mathbb{C P}_{+}^{\infty}\right)\right) \rightarrow \operatorname{Ext}_{M U_{*} M U}^{2, *}\left(M U_{*}, M U_{*}[-4]\right)
$$

where the left-hand side corresponds to the graded abelian group of $M U_{*} M U$ comodule primitives and the right-hand side identifies with the two-line of the AdamsNovikov $E^{2}$-term. The algebraic problem is to determine the image of the algebraic double transfer. To relate this to the original question, it is necessary to understand the stably spherical elements of $M U_{*}\left(\mathbb{C} \mathbf{P}_{+}^{\infty} \wedge \mathbb{C P}_{+}^{\infty}\right)$, namely the comodule primitives

Research partially supported by the project ANR BLAN08-2_338236, HGRT 
in the image of the $M U$-Hurewicz map

$$
\pi_{*}^{\mathrm{s}}\left(\mathbb{C} \mathbf{P}_{+}^{\infty} \wedge \mathbb{C} \mathbf{P}_{+}^{\infty}\right) \rightarrow M U_{*}\left(\mathbb{C} \mathbf{P}_{+}^{\infty} \wedge \mathbb{C} \mathbf{P}_{+}^{\infty}\right)
$$

The latter problem is not addressed here.

The corresponding algebraic framework for the single transfer is well understood by the results of Miller [13]. For the double transfer, the situation is more complicated, since the $M U_{*} M U$-comodule primitives of $M U_{*}\left(\mathbb{C P}_{+}^{\infty} \wedge \mathbb{C} \mathbf{P}_{+}^{\infty}\right)$ are not fully understood and there is additional complexity in passing from the Adams-Novikov one-line to the two-line. Baker approached this algebraic question in [1] by using Morava $K$-theory, working $p$-locally for a prime $p \geq 5$, in particular studying a family of primitives derived from the work of Knapp [10].

This paper studies the algebraic double transfer using invariants that are derived from elliptic homology, Ell, via the $f$-invariant of Laures [12], which requires that 6 is inverted, and via the $f^{\prime}$-invariant of Behrens [4], which is defined when working $p$-locally for $p \geq 5$. For the purposes of this paper, elliptic homology Ell should be taken to be TMF[ $\left.\frac{1}{6}\right]$ (as in [5]), where TMF is the spectrum of topological modular forms. Since the primes 2 and 3 have been inverted, this is a Landweber exact complex oriented theory.

The algebraic double transfer can be studied directly by using the $f$ invariant, which can be considered as an invariant of the comodule primitives of $M U_{*}\left(\mathbb{C} \mathbf{P}_{+}^{\infty} \wedge \mathbb{C} \mathbf{P}_{+}^{\infty}\right)$, taking values in a graded abelian group defined in terms of Katz's ring of divided congruences $\mathfrak{D}$ and the ring of meromorphic modular forms $M F^{\mathrm{mer}}$. The $f$-invariant on elements in the image of the algebraic double transfer is determined by its values on a family of primitives $p_{s} \otimes p_{t} \in M U_{*}\left(\mathbb{C} \mathbf{P}_{+}^{\infty} \wedge \mathbb{C P}_{+}^{\infty}\right)$.

TheOrem 1. For $s, t \in \mathbb{N}$, the $f$-invariant

$$
f\left(p_{s} \otimes p_{t}\right) \in \mathfrak{D}_{\mathbb{Q}} /\left(\mathfrak{D}_{\mathbb{Z}\left[\frac{1}{6}\right]} \oplus\left(M F_{0}^{\mathrm{mer}}\right)_{\mathbb{Q}} \oplus\left(M F_{s+t+2}^{\mathrm{mer}}\right)_{\mathbb{Q}}\right),
$$

is represented by the element $-\bar{B}_{t+1}^{E l l} \bar{B}_{s+1}^{K U} \in \mathfrak{D}_{\mathbb{Q}}$.

Here, $\bar{B}_{*}^{E l l}$ and $\bar{B}_{*}^{K U}$ denote the reduced generalised Bernoulli numbers introduced by Miller (see Section 3.1). This gives an interesting family of elements in the image of the $f$-invariant and sheds light on the comodule primitives, which are detected by the algebraic double transfer.

The remainder of the paper is devoted to showing how to study the algebraic double transfer using the chromatic factorisation of the double transfer

$$
\mathbb{C} \mathbf{P}_{+}^{\infty} \wedge \mathbb{C P}_{+}^{\infty} \rightarrow S^{-4} / p^{\infty}, v_{1}^{\infty}
$$

( $p$-locally for $p \geq 3$ ), which was first constructed by Hilditch (see [2]).

The explicit determination of the induced morphism in $M U$-homology is nontrivial due to the nature of the construction of the chromatic factorisation. It is determined implicitly here by using the Hattori-Stong theorem (see Theorem 3.16 and Proposition 3.17). The conclusion (see Proposition 5.8) is in principle sufficient to be able to calculate the algebraic double transfer on primitive elements; however, identifying the associated classes in $\mathrm{Ext}^{2}$ is non-trivial (compare [1]).

When complex cobordism is replaced by elliptic homology, the $f^{\prime}$-invariant arises naturally when considering the chromatic factorisation as above. The $f^{\prime}$-invariant takes 
values in the comodule primitives of the chromatic comodule $E l l_{*} / p^{\infty}, v_{1}^{\infty}$, which embeds in $E l l_{*} K U \otimes \mathbb{Q} /\left(E l l_{*} K U_{(p)} \oplus\left(E l l_{*}\right)_{\mathbb{Q}}\right)$; composing with this embedding, gives an invariant $f^{\prime \prime}$. In the following statement, $\gamma \in \mathbb{Z}$ denotes a topological generator of the $p$-adic units $\mathbb{Z}_{p}^{\times}$, which is introduced in the construction of the chromatic transfer.

THEOREM 2. The $f^{\prime}$-invariant on the primitives of $E l l_{*}\left(\mathbb{C} \mathbf{P}_{0}^{\infty} \wedge \mathbb{C P}_{0}^{\infty}\right)$ is determined by

$$
f^{\prime \prime}\left(p_{s} \otimes p_{t}\right)=\left[\bar{B}_{s+1}^{E l l} \bar{B}_{t+1}^{K U}+\bar{B}_{s+1}^{K U} \bar{B}_{t+1}^{K U} \frac{\gamma^{s+1}\left(1-\gamma^{t+1}\right)}{\gamma^{s+t+2}-1}\right],
$$

where $s, t \in \mathbb{N}$ and $\bar{B}_{s+1}^{E l l} \bar{B}_{t+1}^{K U}+\bar{B}_{s+1}^{K U} \bar{B}_{t+1}^{K U} \frac{\gamma^{s+1}\left(1-\gamma^{t+1}\right)}{\gamma^{s+t+2}-1}$ is considered as an element of $E l l_{*} K U \otimes \mathbb{Q}$.

Behrens and Laures [5] have established the relationship between the $f$ and $f^{\prime}$ invariants. For the invariants associated to comodule primitives via the double transfer, this relationship is made explicit in Remark 7.11.

2. Chromatic factorisation using $\operatorname{Im}(J)$. This section reviews the techniques for calculating morphisms to the spectrum $L_{1} S / p^{\infty}$ of the chromatic filtration, for $p$ a fixed prime, and how to calculate the induced $M U_{*} M U$-comodule morphisms by using the Hattori-Stong theorem.

The terms ring spectrum and module spectrum refer to the weak, up to homotopy notions. If $E$ is a ring spectrum and $M$ is an $E$-module, the morphism of $E$-module spectra induced by a morphism of spectra $f: X \rightarrow M$ is denoted $\tilde{f}$ : $E \wedge X \rightarrow M$.

2.1. Non-connective $\operatorname{Im}(J)$-theory. Let $\gamma \in \mathbb{Z}$ be a topological generator of the $p$-adic units $\mathbb{Z}_{p}^{\times}$. Non-connective image of $J$-theory, $A d$, is defined by the cofibre sequence

$$
A d \rightarrow K U_{(p)} \stackrel{\psi^{\gamma}-1}{\rightarrow} K U_{(p)} \rightarrow
$$

where $\psi^{\gamma}$ is the stable Adams operation, which is a morphism of ring spectra. The homotopy type of $A d$ is independent of the choice of $\gamma$ (cf. [11]).

The spectrum $A d$ is a $K U$-module spectrum, in particular is $K U$-local; moreover, there are equivalences

$$
A d / p^{\infty} \simeq A d \wedge S / p^{\infty} \simeq\left(L_{1} S\right) \wedge S / p^{\infty} \simeq L_{1}\left(S / p^{\infty}\right)
$$


(cf. [16, Lemma 8.7]), where $L_{1}$ is the Bousfield localisation with respect to $p$-local $K$-theory. Hence, there is a commutative diagram

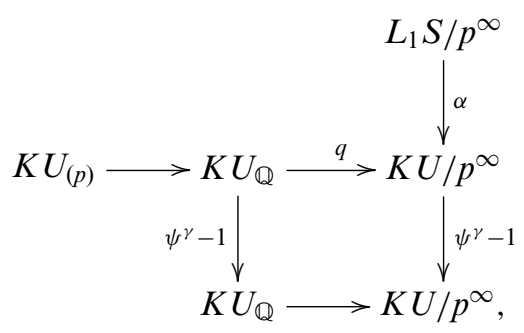

in which the three-term vertical and horizontal sequences are cofibre sequences and $q$ is the reduction morphism. This provides a way of calculating maps to $L_{1} S / p^{\infty}$, as exploited in [2, Section 5] and [8], for example.

For the purposes of this paper, the following terminology is introduced.

Definition 2.1. A $\mathbb{Q}$-representative of a morphism of spectra $g: Y \rightarrow L_{1} S / p^{\infty}$ is a morphism $f: Y \rightarrow K U_{\mathbb{Q}}$ which makes the following diagram commute

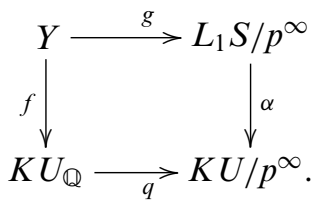

LEMMA 2.2. If $f$ is a $\mathbb{Q}$-representative of $g$, then $\left(\psi^{\gamma}-1\right) f$ lies in the image of $K U_{(p)}^{0} Y \rightarrow K U_{\mathbb{Q}}^{0} Y$.

Proof. Follows from the commutativity of the square in Diagram (1).

PROPOSITION 2.3. Let $Y$ be a spectrum such that $K U_{(p)}^{*} Y$ is a finitely generated free $K U_{(p) *}$-module and $K U_{(p)}^{\text {odd }} Y=0$. Then

(1) the morphism $\left[Y, L_{1} S / p^{\infty}\right] \rightarrow\left[Y, K U / p^{\infty}\right]$ is injective;

(2) any morphism $g: Y \rightarrow L_{1} S / p^{\infty}$ admits a $\mathbb{Q}$-representative;

(3) a morphism $f: Y \rightarrow K U_{\mathbb{Q}}$ such that $\left(\psi^{\gamma}-1\right) f$ lies in the image of $K U_{(p)}^{0} Y \rightarrow$ $K U_{\mathbb{Q}}^{0} Y$ is the $\mathbb{Q}$-representative of a unique morphism $g: Y \rightarrow L_{1} S / p^{\infty}$.

Proof. Straightforward.

EXAmple 2.4. The hypotheses of Proposition 2.3 are satisfied for $Y$ the Thom spectrum of a finite rank virtual $\mathbb{C}$-vector bundle over $\mathbb{C} \mathbf{P}^{n}$ and for smash products of spectra of this type.

2.2. Chromatic factorisation. Recall that a complex oriented ring spectrum $E$ is Landweber exact if the orientation $M U_{*} \rightarrow E_{*}$ is Landweber exact for the Hopf algebroid $\left(M U_{*}, M U_{*} M U\right)$ (see Definition A.10).

LEMMA 2.5. Let E be a Landweber exact complex oriented ring spectrum and $Y$ be a spectrum. 
(1) There exist natural isomorphisms

$$
\operatorname{Hom}_{M U_{*} M U}\left(M U_{*} Y, M U_{*} E\right) \cong \operatorname{Hom}_{M U_{*}}\left(M U_{*} Y, E_{*}\right) \cong \operatorname{Hom}_{E_{*}}\left(E_{*} Y, E_{*}\right) .
$$

(2) For a morphism of spectra $f: Y \rightarrow E$, the comodule morphism $M U_{*} f$ : $M U_{*} Y \rightarrow M U_{*} E$ corresponds via the above isomorphisms to the morphism of $E_{*}$-modules $\tilde{f}_{*}: E_{*} Y \rightarrow E_{*}$ induced by $\tilde{f}: E \wedge Y \rightarrow E$.

Proof. The first isomorphism of part (1) follows from the identification of $M U_{*} E$ as the extended comodule $M U_{*} M U \otimes_{M U_{*}} E_{*}$ and the second follows from the isomorphism of $E_{*}$-modules $E_{*} Y \cong E_{*} \otimes_{M U_{*}} M U_{*} Y$, which is a consequence of Landweber exactness. The final statement is straightforward.

LEMMA 2.6. Let $E$ be a Landweber exact complex oriented ring spectrum, then the morphism $L_{1} S / p^{\infty} \rightarrow K U / p^{\infty}$ induces a monomorphism of $E_{*} E$-comodules, $E_{*} / p^{\infty}\left[v_{1}^{-1}\right] \hookrightarrow E_{*} K U / p^{\infty}$.

Proof. By Landweber exactness, it suffices to prove this result for the universal case $E=M U$, where it is a consequence of the Hattori-Stong theorem (cf. [19, Proposition 20.33]), which states that the $K U-$ Hurewicz morphism $M U_{*} \rightarrow M U_{*} K U$ is rationally faithful (in the terminology of [12, Definition 1.1]), which is equivalent to the statement that $M U_{*} \otimes \mathbb{Q} / \mathbb{Z} \hookrightarrow M U_{*} K U \otimes \mathbb{Q} / \mathbb{Z}$ is a monomorphism. Hence, on the $p$-local component, this gives a monomorphism of $M U_{*} M U$-comodules $M U_{*} / p^{\infty} \hookrightarrow M U_{*} K U / p^{\infty}$.

The morphism of $M U_{*} M U$-comodules $M U_{*} / p^{\infty}\left[v_{1}^{-1}\right] \rightarrow M U_{*} K U / p^{\infty}$ corresponds to the localisation of the above morphism, inverting $v_{1}$, since the morphism $L_{1} S \rightarrow K U_{(p)}$ factors the unit $S \rightarrow K U_{(p)}$. The result follows.

Proposition 2.7. Let $E$ be a Landweber exact complex oriented ring spectrum and $g: Y \rightarrow L_{1} S / p^{\infty}$ be a morphism of spectra which admits a $\mathbb{Q}$-representative $f: Y \rightarrow$ $K U_{\mathbb{Q}}$. Then

(1) the morphism $E_{*}(g): E_{*} Y \rightarrow E_{*}\left(L_{1} S / p^{\infty}\right)$ is determined by $E_{*}(f)$ via the commutative diagram of morphisms of $E_{*} E$-comodules:

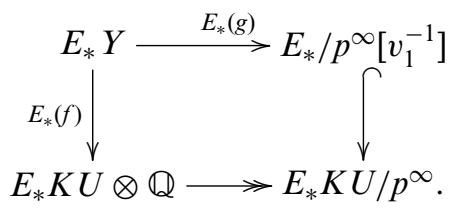

(2) The morphism $E_{*}(f)$ is determined by the morphism of $K U_{*}$-modules $\tilde{f}_{*}$ : $K U_{*} Y \rightarrow K U_{*} \otimes \mathbb{Q}$ induced by $f$.

Proof. Again, by Landweber exactness, it is sufficient to prove the result for the universal case, $E=M U$. 
The commutative Diagram (2) induces a commutative diagram of $M U_{*} M U$ comodules:

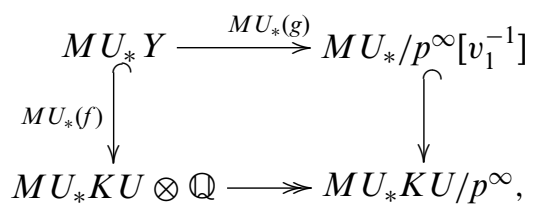

in which $M U_{*} / p^{\infty}\left[v_{1}^{-1}\right] \rightarrow M U_{*} K U / p^{\infty}$ is injective by the Hattori-Stong theorem (Lemma 2.6), and $M U_{*} K U \otimes \mathbb{Q} \rightarrow M U_{*} K U / p^{\infty}$ is the canonical surjection. Thus, $M U_{*}(g)$ is determined by the total composite of the diagram, hence by the morphism of $M U_{*} M U$-comodules $M U_{*} Y \stackrel{M U_{*}(f)}{\rightarrow} M U_{*} K U \otimes \mathbb{Q}$.

The final statement follows from Lemma 2.5, which implies that the morphism $M U_{*}(f)$ is the composite

$$
\begin{aligned}
& M U_{*} Y \quad \stackrel{\psi}{\rightarrow} \quad M U_{*} M U \otimes_{M U_{*}} M U_{*} Y \rightarrow M U_{*} M U \otimes_{M U_{*}} K U_{*} Y \\
& M U_{*} M U \otimes \tilde{f}_{*} M U_{*} K U \otimes \mathbb{Q},
\end{aligned}
$$

where $\psi$ is the comodule structure morphism and the second morphism is induced by $M U_{*} Y \rightarrow K U_{*} Y$, given by the orientation of $K U$.

In the case $E=K U$, this can be made more precise by using the augmentation $K U_{*} K U \rightarrow K U_{*}:$

LEMMA 2.8. Let $g: Y \rightarrow L_{1} S / p^{\infty}$ be a morphism of spectra which admits a $\mathbb{Q}$-representative $f: Y \rightarrow K U_{\mathbb{Q}}$. Then there is an induced commutative diagram of morphisms of $K U_{*}$-modules:

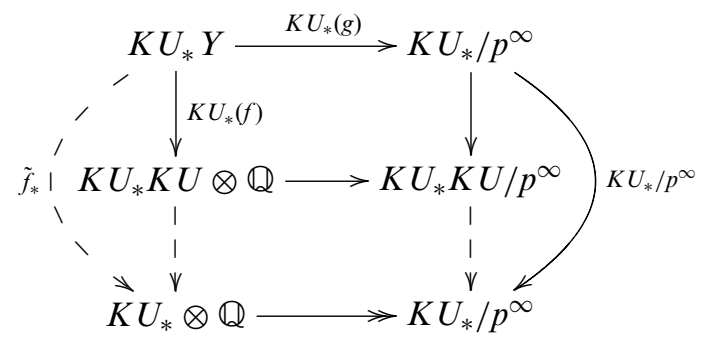

in which the solid arrows are morphisms of $K U_{*} K U$-comodules and the lower vertical morphisms are induced by the augmentation $K U_{*} K U \rightarrow K U_{*}$.

In particular, the comodule morphism $K U_{*}(g): K U_{*} Y \rightarrow K U_{*} / p^{\infty}$ factorises as morphisms of $K U_{*}$-modules as

$$
K U_{*} Y \stackrel{\tilde{f}_{*}}{\rightarrow} K U_{*} \otimes \mathbb{Q} \rightarrow K U_{*} / p^{\infty} .
$$

3. Chromatic factorisation of the double transfer. This section reviews the construction of the chromatic factorisation of the double transfer (see Theorem 3.12), working $p$-locally at an odd prime $p$. The morphism in $M U_{*}$-homology is calculated 
implicitly in $M U_{*} M U$-comodules (see Theorem 3.16) by applying the results of Section 2.

3.1. Generalised Bernoulli numbers. To fix notation, recall that the Hopf algebroid $\left(M U_{*}, M U_{*} M U\right)$ is isomorphic to the Hopf algebroid $(L, L B)$, which represents the groupoid scheme of formal group laws and strict isomorphisms, where $L$ is the Lazard ring and $L B \cong L\left[b_{i} \mid i \geq 0, b_{0}=1\right]$ as a left $L$-algebra (cf. [17]). The $b_{i}^{\prime} s$ represent the universal strict isomorphism $\underline{b}(x)=\sum_{i} b_{i} x^{i+1}$ between the formal group laws defined respectively by the left and right units $\eta_{L}, \eta_{R}: L \rightrightarrows L B$, which are determined by their logarithms $\log ^{L}, \log ^{R}$ defined over $L B \otimes \mathbb{Q}$. The exponential series $\exp ^{L}, \exp ^{R}$ over $L B \otimes \mathbb{Q}$ are the respective composition inverses of $\log ^{L}$ and $\log ^{R}$.

LEMMA 3.1. The power series $\underline{b}$ satisfies the identity $\underline{b}=\exp ^{R} \circ \log ^{L}$.

Definition 3.2. [13, Definition 1.1] Let $F$ be a formal group law defined over a ring $R$. The Bernoulli numbers $B_{n}(F) \in R \otimes \mathbb{Q}$, for strictly positive integers $n \in \mathbb{Z}_{>0}$, are defined by

$$
\frac{1}{\exp ^{F} x}-\frac{1}{x}=\sum_{i \geq 0} \frac{B_{i+1}(F)}{(i+1) !} x^{i},
$$

where $\exp ^{F}(x) \in(R \otimes \mathbb{Q})[[x]]$ is the exponential of $F$. The reduced Bernoulli number $\bar{B}_{n}(F)$ is defined as $\bar{B}_{n}(F):=\frac{B_{n}(F)}{n} \in R \otimes \mathbb{Q}$.

EXAMPLE 3.3. For $n \in \mathbb{Z}_{>0}$, write $B_{n}^{K U} \in K U_{*} \otimes \mathbb{Q}$ (respectively $\bar{B}_{n}^{K U} \in K U_{*} \otimes \mathbb{Q}$ ) for the Bernoulli number (respectively reduced Bernoulli number) associated to the orientation of $K U$. This is a graded form of the usual Bernoulli number $B_{n}$ (respectively reduced).

REMARK 3.4. If the formal group law $F$ is graded with respect to the usual conventions (so that the coordinate has degree -2 ), then $B_{n}(F)$ is a homogeneous element of degree $2 n$.

REMARK 3.5. Miller established the following fundamental divisibility property of the reduced Bernoulli numbers: if $R$ is a torsion-free $\operatorname{ring}$, then $d_{n} \bar{B}_{n}(F) \in R$, where $d_{n}$ is the order of the reduced Bernoulli number $\bar{B}_{n}$ in $\mathbb{Q} / \mathbb{Z}$ (see [13, Theorem 1.3]).

\subsection{The single $S^{1}$-transfer.}

Definition 3.6. For $n \in \mathbb{Z}$, let $\mathbb{C} \mathbf{P}_{n}^{\infty}$ denote the Thom spectrum of the (virtual) bundle $n \lambda$ over $\mathbb{C} \mathbf{P}^{\infty}$, where $\lambda$ denotes the canonical line bundle over $\mathbb{C} \mathbf{P}^{\infty}$.

For $E$ a complex oriented ring spectrum, the Thom isomorphism implies that $E_{*}\left(\mathbb{C} \mathbf{P}_{n}^{\infty}\right)$ is a free $E_{*}$-module on classes $\left\{\beta_{i} \mid i \geq n\right\}$. (The systems of generators as $n$ varies are compatible, hence $n$ will be omitted from the notation.) There is a Künneth isomorphism $E_{*}\left(\mathbb{C} \mathbf{P}_{m}^{\infty} \wedge \mathbb{C} \mathbf{P}_{n}^{\infty}\right) \cong E_{*}\left(\mathbb{C} \mathbf{P}_{m}^{\infty}\right) \otimes_{E_{*}} E_{*}\left(\mathbb{C} \mathbf{P}_{n}^{\infty}\right)$, and the associated module generators will be written $\beta_{i} \otimes \beta_{j}$.

NotATION 3.7. For $E$ a complex oriented ring spectrum and $m, n$ integers, let $\underline{\beta}_{m}(S)$ denote the Laurent power series $\sum_{i \geq m} \beta_{i} S^{i}$ over $E_{*}\left(\mathbb{C} \mathbf{P}_{m}^{\infty}\right)$ and let $\underline{\beta}_{m}(S) \otimes \underline{\beta}_{n}(T)$ denote $\sum_{i \geq m, j \geq n} \beta_{i} \otimes \beta_{j} S^{i} T^{j}$, defined over $E_{*}\left(\mathbb{C} \mathbf{P}_{m}^{\infty} \wedge \mathbb{C} \mathbf{P}_{n}^{\infty}\right)$. 
Such generating power series provide an efficient way of encoding calculations. For example:

LEMma 3.8. [13, Proposition 3.3] Let $n$ be an integer, then the comodule structure $M U_{*}\left(\mathbb{C} \mathbf{P}_{n}^{\infty}\right) \rightarrow M U_{*} M U \otimes_{M U_{*}} M U_{*}\left(\mathbb{C P}_{n}^{\infty}\right)$ is determined by

$$
\underline{\beta}_{n}(S) \mapsto \underline{\beta}_{n}(\underline{b}(S) \otimes 1)
$$

REMARK 3.9. In the expression $\underline{\beta}_{n}(\underline{b}(x) \otimes 1)$, the elements $\beta_{i}$ are the module generators, which are usually written ${ }^{n}$ on the right when considering left $M U_{*} M U$ comodules. Miller [13] works with right comodules, where this notational issue does not arise.

The cofibre sequence of spectra (cf. [13, Section 2]):

$$
S^{2 n} \rightarrow \mathbb{C} \mathbf{P}_{n}^{\infty} \rightarrow \mathbb{C} \mathbf{P}_{n+1}^{\infty} \rightarrow
$$

for $n \in \mathbb{Z}$, induces a short exact sequence of $M U_{*} M U$-comodules:

$$
0 \rightarrow M U_{*}[2 n] \rightarrow M U_{*}\left(\mathbb{C P}_{n}^{\infty}\right) \rightarrow M U_{*}\left(\mathbb{C P}_{n+1}^{\infty}\right) \rightarrow 0
$$

where $[a]$ denotes the shift in degree so that $\left(V_{*}[a]\right)_{t}=V_{t-a}$, for a $\mathbb{Z}$-graded object $V_{*}$.

The choice of generators gives a standard splitting of this sequence in $M U_{*}$ modules. In particular:

Notation 3.10. For E a complex oriented ring spectrum,

(1) let $\sigma: E_{*}\left(\mathbb{C} \mathbf{P}_{0}^{\infty}\right) \rightarrow E_{*}\left(\mathbb{C} \mathbf{P}_{-1}^{\infty}\right)$ be the section in $E_{*}$-modules defined by $\sigma\left(\beta_{i}\right)=\beta_{i}$ (for $i \geq 0$ ) and $r: E_{*}\left(\mathbb{C P}_{-1}^{\infty}\right) \rightarrow E_{*}[-2]$ be the corresponding retract, which sends generators $\beta_{i}, i \geq 0$ to zero.

(2) let $\sigma^{\prime}: E_{*}\left(\mathbb{C P}_{0}^{\infty} \wedge \mathbb{C} \mathbf{P}_{0}^{\infty}\right) \rightarrow E_{*}\left(\mathbb{C} \mathbf{P}_{-1}^{\infty} \wedge \mathbb{C} \mathbf{P}_{0}^{\infty}\right)$ denote the section $\sigma \otimes E_{*}\left(\mathbb{C} \mathbf{P}_{0}^{\infty}\right)$.

For $n=-1$, the connecting morphism of the cofibre sequence (3) defines the $S^{1}$-transfer $\tau: \mathbb{C P}_{0}^{\infty} \rightarrow S^{-1}$. The double $S^{1}$-transfer is the smash product $\tau \wedge \tau$ : $\mathbb{C} \mathbf{P}_{0}^{\infty} \wedge \mathbb{C} \mathbf{P}_{0}^{\infty} \rightarrow S^{-2}$.

The rational Thom class $U: \mathbb{C} \mathbf{P}_{-1}^{\infty} \rightarrow S_{\mathbb{Q}}^{-2}$ induces a morphism of cofibre sequences

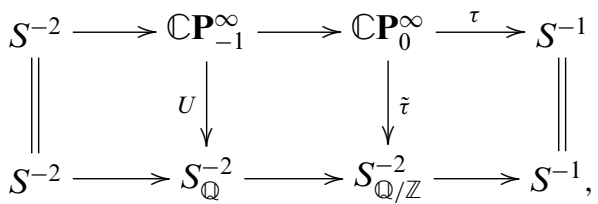

where $\tilde{\tau}$, the chromatic factorisation of the single transfer, is determined uniquely by the commutativity of the right-hand square.

The morphism of $M U_{*} M U$-comodules

$$
M U_{*}(\tilde{\tau}): M U_{*}\left(\mathbb{C} \mathbf{P}_{0}^{\infty}\right) \rightarrow M U_{*} \otimes \mathbb{Q} / \mathbb{Z}[-2]
$$


is determined by the comodule morphism $M U_{*}(U): M U_{*}\left(\mathbb{C} \mathbf{P}_{-1}^{\infty}\right) \rightarrow M U_{*} \otimes \mathbb{Q}[-2]$ via the commutative diagram

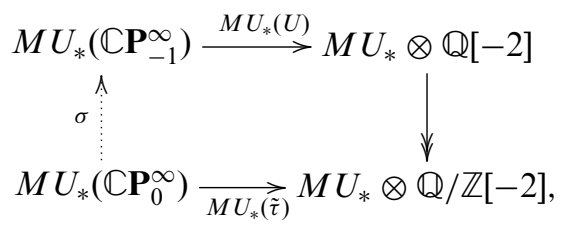

in which the solid arrows denote comodule morphisms.

By Lemma 2.5, the comodule morphism $M U_{*}(U)$ is the composite

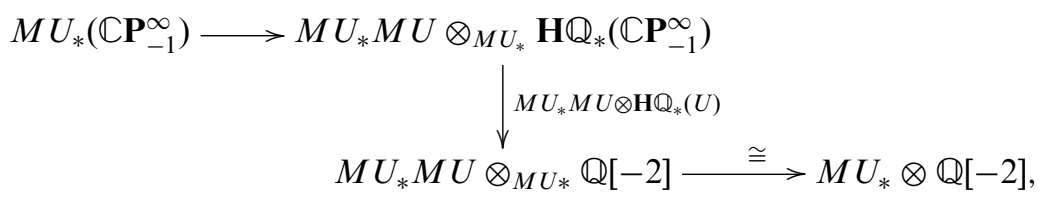

where $\mathbf{H} \mathbb{Q}$ is the rational Eilenberg-MacLane spectrum and the first morphism is the composite of the comodule structure morphism with $M U_{*}\left(\mathbb{C} \mathbf{P}_{-1}^{\infty}\right) \rightarrow \mathbf{H} \mathbb{Q}_{*}\left(\mathbb{C} \mathbf{P}_{-1}^{\infty}\right)$ induced by the canonical orientation of $\mathbf{H} \mathbb{Q}$.

LEMMA 3.11. (Cf. [13, Theorem 3.9])

(1) The morphism $M U_{*}(U): M U_{*}\left(\mathbb{C} \mathbf{P}_{-1}^{\infty}\right) \rightarrow M U_{*} \otimes \mathbb{Q}[-2]$ is determined by

$$
\underline{\beta}_{-1}(S) \mapsto \frac{1}{\log S} .
$$

(2) The morphism of $M U_{*}$-modules $M U_{*}(U) \circ \sigma: M U_{*}\left(\mathbb{C P}_{0}^{\infty}\right) \rightarrow M U_{*} \otimes \mathbb{Q}[-2]$ is determined by

$$
\underline{\beta}_{0}(S) \mapsto \frac{1}{\log S}-\frac{1}{S} .
$$

(3) The morphism $M U_{*}(\tilde{\tau})$ is the composite of $M U_{*}(U) \circ \sigma$ with the projection $M U_{*} \otimes \mathbb{Q}[-2] \rightarrow M U_{*} \otimes \mathbb{Q} / \mathbb{Z}[-2]$.

Proof. The first statement follows from the comodule structure of $M U_{*}\left(\mathbb{C} \mathbf{P}_{-1}^{\infty}\right)$ together with the fact that, under the morphism $M U_{*} M U \otimes \mathbb{Q} \cong M U_{*} \otimes M U_{*} \otimes$ $\mathbb{Q} \rightarrow M U_{*} \otimes \mathbb{Q}$ induced by the augmentation $M U_{*} \otimes \mathbb{Q} \rightarrow \mathbb{Q}$ on the right-hand factor, $\exp ^{R}(S) \mapsto S$, so that $\underline{b}(S) \mapsto \log (S)$.

The section $\sigma$ is determined by $\underline{\beta}_{0}(S) \mapsto \underline{\beta}_{-1}(S)-\beta_{-1} \frac{1}{S}$, which gives the second statement, by composition. The final statement follows from the commutativity of Diagram (5).

3.3. The chromatic factorisation of the double transfer. Working $p$-locally ( $p$ odd), the above chromatic factorisation of the single transfer extends to a chromatic factorisation of the double transfer, for which the original published reference is [2, Theorem 5.2], where the result is attributed to Hilditch, and a generalisation is given by Imaoka in [8]. (Imaoka [7] has also considered the chromatic factorisation of the double transfer at the prime $p=2$.) 
THEOREM 3.12. [2] Let $p \geq 3$ be a prime. There exists a morphism $\Theta$ : $\mathbb{C} \mathbf{P}_{-1}^{\infty} \wedge \mathbb{C P}_{0}^{\infty} \rightarrow L_{1} S^{-4} / p^{\infty}$ which fits into a commutative square:

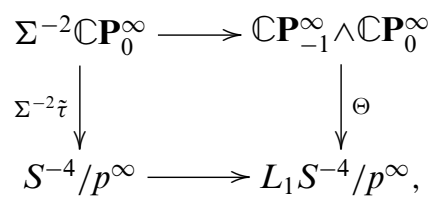

where the top morphism is induced by the inclusion of the bottom cell $S^{-2} \rightarrow \mathbb{C} \mathbf{P}_{-1}^{\infty}$.

Moreover, for any extension to a morphism of cofibre sequences:

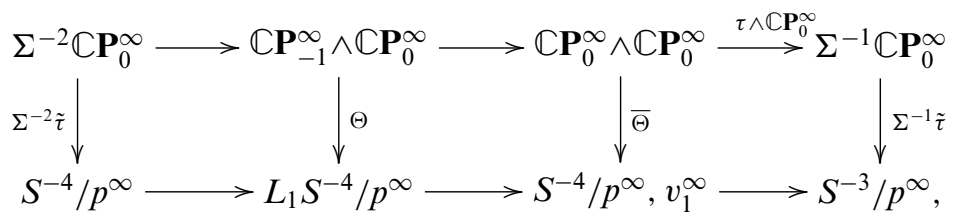

where the top row is the cofibre sequence $\left(S^{-2} \rightarrow \mathbb{C} \mathbf{P}_{-1}^{\infty} \rightarrow \mathbb{C} \mathbf{P}_{0}^{\infty}\right) \wedge \mathbb{C} \mathbf{P}_{0}^{\infty}$ and the bottom row is the cofibre sequence associated to $L_{1}$-localisation, $\bar{\Theta}: \mathbb{C} \mathbf{P}_{0}^{\infty} \wedge \mathbb{C} \mathbf{P}_{0}^{\infty} \rightarrow S^{-4} / p^{\infty}, v_{1}^{\infty}$ provides a factorisation of the double transfer morphism across the chromatic morphism $S^{-4} / p^{\infty}, v_{1}^{\infty} \rightarrow S^{-2}$.

The morphism $\Theta$ is constructed using Proposition 2.3, by defining an explicit cohomology class cohomology class $\theta \in\left[\mathbb{C} \mathbf{P}_{-1}^{\infty} \wedge \mathbb{C P}_{0}^{\infty}, \Sigma^{-4} K U_{\mathbb{Q}}\right]$ such that there is a commutative diagram

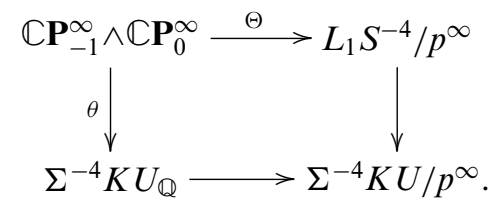

REMARK 3.13. The construction of $\theta$ is by an eigenspace argument for the action of the Adams operation $\psi^{\gamma}$, where $\gamma \in \mathbb{Z}$ is a topological generator of $\mathbb{Z}_{p}$ (compare Proposition 2.3); this is made explicit in the proof of [8, Proposition 2.4], which generalises this result.

For later use, the following notation is introduced.

NotATION 3.14. Let $\tilde{\theta}^{\prime}(S, T)$ denote the power series in $K U_{*} \otimes \mathbb{Q}[[S, T]]$

$$
\sum_{i, j>0} \frac{B_{i}^{K U}}{i !} \frac{B_{j}^{K U}}{j !}\left(\frac{\gamma^{i}-1}{\gamma^{i+j}-1}\right)\left(\log ^{K U} S\right)^{i-1}\left(\log ^{K U} T\right)^{j-1},
$$

where $\log ^{K U}$ is the logarithm of the multiplicative formal group law of $K U_{*} \otimes \mathbb{Q}$.

The morphism $\theta$ is not uniquely defined; [2, Theorem 5.2] gives an explicit choice for $\theta$, working with the $p$-local Adams summand $E(1)$, which can be replaced by $p$-local $K$-theory. The following choice is used here: 
Definition 3.15. Let $\theta: \mathbb{C P}_{-1}^{\infty} \wedge \mathbb{C P}_{0}^{\infty} \rightarrow \Sigma^{-4} K U_{\mathbb{Q}}$ be the class which is determined by $\tilde{\theta}_{*}: K U_{*}\left(\mathbb{C} \mathbf{P}_{-1}^{\infty} \wedge \mathbb{C} \mathbf{P}_{0}^{\infty}\right) \rightarrow K U_{*} \otimes \mathbb{Q}[-4]$ :

$$
\underline{\beta}_{-1}(S) \otimes \underline{\beta}_{0}(T) \mapsto \frac{1}{S}\left(\frac{1}{\log ^{K U} T}-\frac{1}{T}\right)+\tilde{\theta}^{\prime}(S, T) .
$$

3.4. Calculating in complex cobordism. Let $p$ be a fixed odd prime and $\Theta, \bar{\Theta}$ be as in Theorem 3.12, where the $\mathbb{Q}$-representative $\theta$ of $\Theta$ is the morphism of Definition 3.15 .

THEOREM 3.16. Let p be an odd prime. There is a commutative diagram of morphisms of $M U_{*} M U$-comodules:

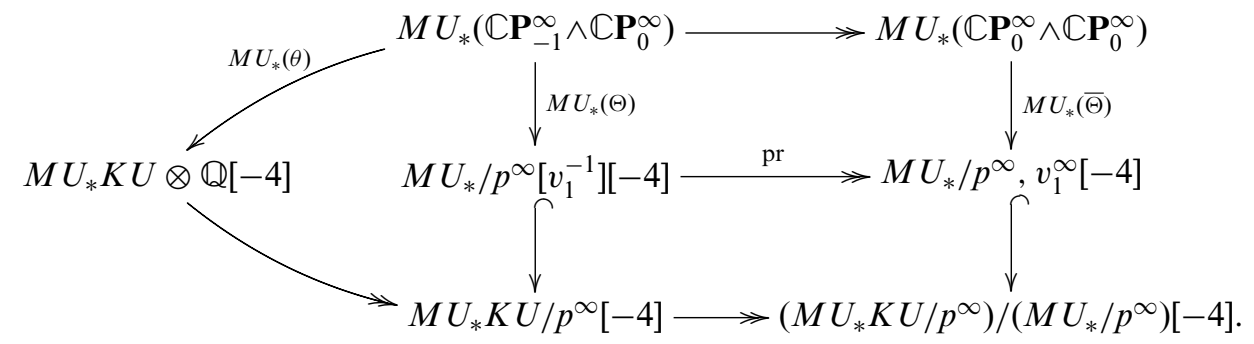

(1) The underlying $M U_{*}$-module morphism of $M U_{*}(\bar{\Theta})$ is the composite pro $M U_{*}(\Theta) \circ \sigma^{\prime}$.

(2) The morphism $M U_{*}(\Theta)$ is determined by the comodule morphism $M U_{*}(\theta)$ and hence by the morphism $\tilde{\theta}_{*}: K U_{*}\left(\mathbb{C} \mathbf{P}_{-1}^{\infty} \wedge \mathbb{C P}_{0}^{\infty}\right) \rightarrow K U_{*} \otimes \mathbb{Q}$.

(3) The morphism $M U_{*}(\bar{\Theta})$ is determined by the comodule morphism $M U_{*}(\theta)$.

Proof. The commutativity of the left-hand part of the diagram follows from Proposition 2.7 and the upper right-hand square is induced by the morphism of cofibre sequences defining $\bar{\Theta}$. The lower right-hand square is induced by the monomorphism given by the Hattori-Stong theorem (see Lemma 2.6)

$$
M U_{*} / p^{\infty}\left[v_{1}^{-1}\right] \hookrightarrow M U_{*} K U / p^{\infty},
$$

since the kernel to the projection $M U_{*} / p^{\infty}\left[v_{1}^{-1}\right] \rightarrow M U_{*} / p^{\infty}, v_{1}^{\infty}$ is $M U_{*} / p^{\infty}$.

The morphism $M U_{*}\left(\mathbb{C} \mathbf{P}_{-1}^{\infty} \wedge \mathbb{C} \mathbf{P}_{0}^{\infty}\right) \rightarrow M U_{*}\left(\mathbb{C P}_{0}^{\infty} \wedge \mathbb{C} \mathbf{P}_{0}^{\infty}\right)$ admits the section $\sigma^{\prime}$ in $M U_{*}$-modules, hence the identification of the underlying module morphism of $M U_{*}(\bar{\Theta})$ follows from the upper right-hand square.

For part 2, the injectivity of $M U_{*} / p^{\infty}, v_{1}^{\infty}[-4] \hookrightarrow M U_{*} K U / p^{\infty}[-4]$ and the commutativity of the left-hand square implies that $M U_{*}(\theta)$ determines $M U_{*}(\Theta)$ as a morphism of $M U_{*} M U$-comodules. The morphism $M U_{*}(\theta)$ is determined by $\tilde{\theta}_{*}: K U_{*}\left(\mathbb{C} \mathbf{P}_{-1}^{\infty} \wedge \mathbb{C} \mathbf{P}_{0}^{\infty}\right) \rightarrow K U_{*} \otimes \mathbb{Q}$ by the second part of Proposition 2.7.

The final statement follows from the commutativity of the lower right-hand square.

3.5. Calculating $M U_{*}(\theta)$. The torsion-free ring $M U_{*} K U$ has two formal group law structures, corresponding to the left and right units $M U_{*} \rightarrow M U_{*} K U$ and $K U_{*} \rightarrow$ 
$M U_{*} K U$ respectively; write $\log ^{M U}$ and $\log ^{K U}$ for the respective logarithms defined over $M U_{*} K U \otimes \mathbb{Q}$ and set $\underline{b}^{\prime}=\exp ^{K U} \circ \log ^{M U}$, which identifies with the image of $\underline{b}$ under $M U_{*} M U \rightarrow M U_{*} K U$.

Proposition 3.17.

(1) The morphism $M U_{*}(\theta) \in \operatorname{Hom}_{M U_{*} M U}\left(M U_{*}\left(\mathbb{C P}_{-1}^{\infty} \wedge \mathbb{C} \mathbf{P}_{0}^{\infty}\right), M U_{*} K U \otimes \mathbb{Q}[-4]\right)$ is determined by $\underline{\beta}_{-1}(S) \otimes \underline{\beta}_{0}(T) \mapsto$

$$
\begin{aligned}
& \frac{1}{\underline{b}^{\prime}(S)}\left(\frac{1}{\log ^{M U} T}-\frac{1}{\underline{b}^{\prime}(T)}\right) \\
& \quad+\sum_{i, j>0} \frac{B_{i}^{K U}}{i !} \frac{B_{j}^{K U}}{j !}\left(\frac{\gamma^{i}-1}{\gamma^{i+j}-1}\right)\left(\log ^{M U} S\right)^{i-1}\left(\log ^{M U} T\right)^{j-1}
\end{aligned}
$$

(2) The morphism $M U_{*}(\theta) \circ \sigma^{\prime} \in \operatorname{Hom}_{M U_{*}}\left(M U_{*}\left(\mathbb{C P}_{0}^{\infty} \wedge \mathbb{C P}_{0}^{\infty}\right), M U_{*} K U \otimes\right.$ $\mathbb{Q}[-4])$ is determined by $\underline{\beta}_{0}(S) \otimes \underline{\beta}_{0}(T) \mapsto$

$$
\begin{aligned}
& \left(\frac{1}{\underline{b}^{\prime}(S)}-\frac{1}{S}\right)\left(\frac{1}{\log ^{M U} T}-\frac{1}{\underline{b}^{\prime}(T)}\right) \\
& \quad+\sum_{i, j>0} \frac{B_{i}^{K U}}{i !} \frac{B_{j}^{K U}}{j !}\left(\frac{\gamma^{i}-1}{\gamma^{i+j}-1}\right)\left(\log ^{M U} S\right)^{i-1}\left(\log ^{M U} T\right)^{j-1}
\end{aligned}
$$

Proof. By Proposition 2.7, the morphism $M U_{*}(\theta)$ is determined by the commutative diagram

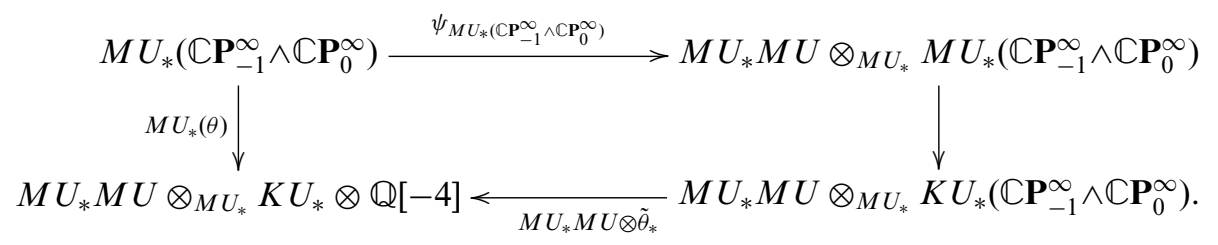

Lemma 3.8 implies that the comodule structure of $M U_{*}\left(\mathbb{C} \mathbf{P}_{-1}^{\infty} \wedge \mathbb{C P}_{0}^{\infty}\right)$ is determined by

$$
\underline{\beta}_{-1}(S) \otimes \underline{\beta}_{0}(T) \mapsto \underline{\beta}_{-1}(\underline{b}(S) \otimes 1) \otimes \underline{\beta}_{0}(\underline{b}(T) \otimes 1) .
$$

Composing with the morphism induced by $\theta$ and using the identity $\underline{b}^{\prime}=\exp ^{K U} \circ \log ^{M U}$ shows that $M U_{*}(\theta)$ is given by $\underline{\beta}_{-1}(S) \otimes \underline{\beta}_{0}(T) \mapsto$

$$
\frac{1}{\underline{b}^{\prime}(S)}\left(\frac{1}{\log ^{M U} T}-\frac{1}{\underline{b}^{\prime}(T)}\right)+\sum_{i, j>0} \frac{B_{i}^{K U}}{i !} \frac{B_{j}^{K U}}{j !}\left(\frac{\gamma^{i}-1}{\gamma^{i+j}-1}\right)\left(\log ^{M U} S\right)^{i-1}\left(\log ^{M U} T\right)^{j-1} .
$$

The second statement is proved by composing with the morphism $\sigma^{\prime}$, which is represented by

$$
\underline{\beta}_{0}(S) \otimes \underline{\beta}_{0}(T) \mapsto \underline{\beta}_{-1}(S) \otimes \underline{\beta}_{0}(T)-\beta_{-1} \frac{1}{S} \otimes \underline{\beta}_{0}(T) .
$$


The morphism $M U_{*}(\theta)$ restricts to give

$$
\beta_{-1} \otimes \underline{\beta}_{0}(T) \mapsto \frac{1}{\log ^{M U} T}-\frac{1}{\underline{b}^{\prime}(T)} .
$$

The result follows.

4. The algebraic transfer. The algebraic version of the double transfer is introduced in this section and is related to chromatic theory.

4.1. The algebraic transfer. The cofibre sequence defining the transfer $\tau$ : $\mathbb{C} \mathbf{P}_{0}^{\infty} \rightarrow S^{-1}$ induces a short exact sequence of $M U_{*} M U$-comodules and hence an algebraic transfer class:

$$
\left[e_{\tau}\right] \in \operatorname{Ext}_{M U_{*} M U}^{1}\left(M U_{*}\left(\mathbb{C P}_{0}^{\infty}\right), M U_{*}[-2]\right) .
$$

Definition 4.1. The algebraic double transfer is the class:

$$
\left[e_{\tau}\right]^{2} \in \operatorname{Ext}_{M U_{*} M U}^{2}\left(M U_{*}\left(\mathbb{C} \mathbf{P}_{0}^{\infty} \wedge \mathbb{C} \mathbf{P}_{0}^{\infty}\right), M U_{*}[-4]\right)
$$

given by the Yoneda product.

Proposition A.8 applied with respect to the section $\sigma$ gives the standard choice $e_{\tau}$ of representing cocycle: by

LEMMA 4.2. The cocycle $e_{\tau} \in \operatorname{Hom}_{M U_{*}}\left(M U_{*}\left(\mathbb{C P}_{0}^{\infty}\right), M U_{*} M U[-2]\right)$ is determined

$$
\underline{\beta}_{0}(S) \mapsto \frac{1}{S}-\frac{1}{\underline{b}(S)}
$$

Diagram (4) induces a morphism of short exact sequences of $M U_{*} M U$-comodules:

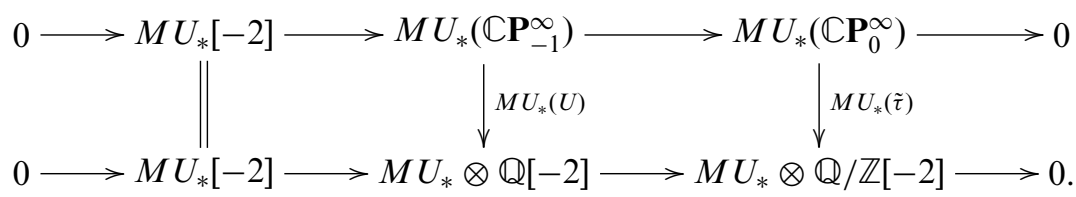

The morphism $\tilde{\tau}$ provides a chromatic factorisation of the single transfer $\tau$; this corresponds to the following result, which can be proved using Proposition A.8.

Proposition 4.3. There is an equality in $\operatorname{Ext}_{M U_{*} M U}^{1}\left(M U_{*}\left(\mathbb{C} \mathbf{P}_{0}^{\infty}\right), M U_{*}[-2]\right)$ :

$$
\left[e_{\tau}\right]=\partial_{1} M U_{*}(\tilde{\tau})
$$

where $\partial_{1}$ is the chromatic connecting morphism associated to

$$
0 \longrightarrow M U_{*}[-2] \longrightarrow M U_{*} \otimes \mathbb{Q}[-2] \longrightarrow M U_{*} \otimes \mathbb{Q} / \mathbb{Z}[-2] \longrightarrow 0 .
$$


4.2. The class $[\kappa]$. Rather than working directly with the double algebraic transfer $\left[e_{\tau}\right]^{2}$, it is convenient to work with a class $[\kappa]$ in $\operatorname{Ext}^{1}$ (see Definition 4.5), which is related to the double transfer via the chromatic connecting morphism $\partial_{1}$ (see Proposition 4.6).

Forming the tensor product $\left[e_{\tau}\right] \otimes M U_{*}\left(\mathbb{C} \mathbf{P}_{0}^{\infty}\right)$ gives a class

$$
\left[E_{\tau}\right] \in \operatorname{Ext}_{M U_{*} M U}^{1}\left(M U_{*}\left(\mathbb{C P}_{0}^{\infty} \wedge \mathbb{C P}_{0}^{\infty}\right), M U_{*}\left(\mathbb{C P}_{0}^{\infty}\right)[-2]\right) .
$$

LEMMA 4.4. The class $\left[E_{\tau}\right]$ is represented by the cocycle

$$
E_{\tau} \in \operatorname{Hom}_{M U_{*}}\left(M U_{*}\left(\mathbb{C P}_{0}^{\infty} \wedge \mathbb{C P}_{0}^{\infty}\right), M U_{*} M U \otimes_{M U_{*}} M U_{*}\left(\mathbb{C} \mathbf{P}_{0}^{\infty}\right)[-2]\right)
$$

defined with respect to the section $\sigma^{\prime}$, which is given by

$$
\underline{\beta}_{0}(S) \otimes \underline{\beta}_{0}(T) \mapsto\left(\frac{1}{S}-\frac{1}{\underline{b}(S)}\right) \underline{\beta}_{0}(\underline{b}(T)) .
$$

Proof. Apply Proposition A.8 with respect to the section $\sigma^{\prime}$.

DEFINITION 4.5. Let $[\kappa]$ denotes the class

$$
M U_{*}(\tilde{\tau})\left[E_{\tau}\right] \in \operatorname{Ext}_{M U_{*} M U}^{1}\left(M U_{*}\left(\mathbb{C} \mathbf{P}_{0}^{\infty} \wedge \mathbb{C P}_{0}^{\infty}\right), M U_{*} \otimes \mathbb{Q} / \mathbb{Z}[-4]\right) .
$$

The following result justifies using $\kappa$ in place of the algebraic double transfer.

Proposition 4.6. There is an identity

$$
\left[e_{\tau}\right]^{2}=\partial_{1}[\kappa],
$$

in $\operatorname{Ext}_{M U_{*} M U}^{2}\left(M U_{*}\left(\mathbb{C P}_{0}^{\infty} \wedge \mathbb{C P}_{0}^{\infty}\right), M U_{*}[-4]\right)$.

Proof. Straightforward.

The class $[\kappa]$ is represented by the standard choice $\kappa$ of cocycle, constructed with respect to the section $\sigma^{\prime}$ :

$$
\kappa \in \operatorname{Hom}_{M U_{*}}\left(M U_{*}\left(\mathbb{C P}_{0}^{\infty} \wedge \mathbb{C P}_{0}^{\infty}\right), M U_{*} M U \otimes \mathbb{Q} / \mathbb{Z}[-4]\right) .
$$

Notation 4.7. Write $K \in \operatorname{Hom}_{M U_{*}}\left(M U_{*}\left(\mathbb{C P}_{0}^{\infty} \wedge \mathbb{C P}_{0}^{\infty}\right), M U_{*} M U \otimes \mathbb{Q}[-4]\right)$ for the composite morphism of $M U_{*}$-modules:

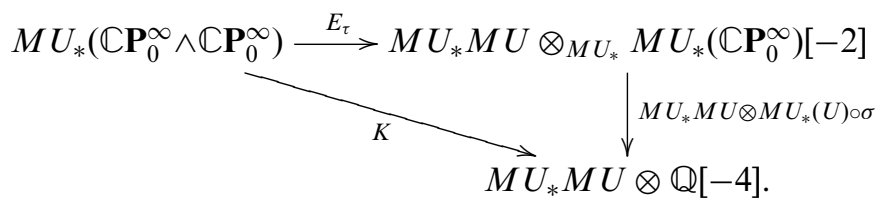

PROPOSITION 4.8.

(1) The class $[\kappa]$ is represented by the cocycle $\kappa$ given by reduction of the morphism $K$ via $M U_{*} M U \otimes \mathbb{Q}[-4] \rightarrow M U_{*} M U \otimes \mathbb{Q} / \mathbb{Z}[-4]$. 
(2) The morphism $K \in \operatorname{Hom}_{M U_{*}}\left(M U_{*}\left(\mathbb{C P}_{0}^{\infty} \wedge \mathbb{C P}_{0}^{\infty}\right), M U_{*} M U \otimes \mathbb{Q}[-4]\right)$ is determined by

$$
\underline{\beta}_{0}(S) \otimes \underline{\beta}_{0}(T) \mapsto\left(\frac{1}{S}-\frac{1}{\underline{b}(S)}\right)\left(\frac{1}{\log ^{L} T}-\frac{1}{\underline{b}(T)}\right) .
$$

Proof. The first statement follows from the construction of $K$.

The second follows from Lemma 4.4, by composition with the morphism $M U_{*} M U \otimes\left(M U_{*}(U) \circ \sigma\right)$ (see Lemma 3.11), using the identity $\log ^{L}=\log ^{R} \circ \underline{b}$ of Lemma 3.1.

4.3. Relation with the chromatic factorisation of the double transfer. Let $p$ be an odd prime. Here $\kappa$ will be written to denote the associated $p$-local cocycle:

$$
\kappa \in \operatorname{Hom}_{M U_{*}}\left(M U_{*}\left(\mathbb{C} \mathbf{P}_{0}^{\infty} \wedge \mathbb{C P}_{0}^{\infty}\right), M U_{*} M U / p^{\infty}[-4]\right) .
$$

The construction of $\bar{\Theta}$ as a chromatic factorisation of the double transfer implies that the class $[\kappa]$ is related to the morphism $M U_{*}(\bar{\Theta})$. Write $\partial_{2}$ for the chromatic connecting morphism associated to the short exact sequence of comodules

$$
0 \rightarrow M U_{*} / p^{\infty} \rightarrow M U_{*} / p^{\infty}\left[v_{1}^{-1}\right] \rightarrow M U_{*} / p^{\infty}, v_{1}^{\infty} \rightarrow 0
$$

Proposition 4.9. There is an identity $[\kappa]=\partial_{2} M U_{*}(\bar{\Theta})$ in $\operatorname{Ext}_{M U_{*} M U}^{1}\left(M U_{*}\left(\mathbb{C P}_{0}^{\infty} \wedge \mathbb{C P}_{0}^{\infty}\right), M U_{*} / p^{\infty}[-4]\right)$. In particular, $\partial_{2} M U_{*}(\bar{\Theta})$ is independent of the choice of $\bar{\Theta}$.

Proof. The morphism $M U_{*}(\Theta)$ gives rise to a morphism between short exact sequences of $M U_{*} M U$-comodules:

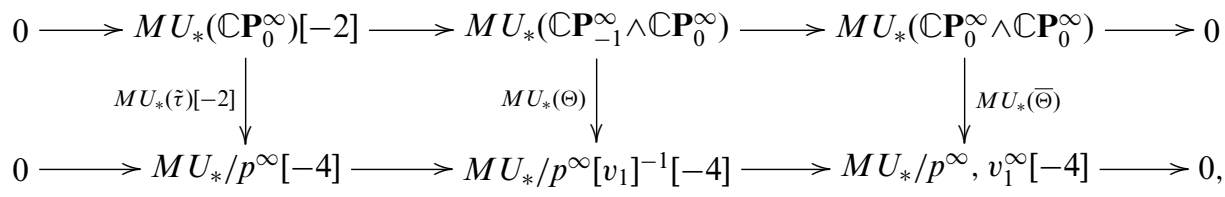

where the top row represents $\left[E_{\tau}\right]$. By definition, $[\kappa]=M U_{*}(\tilde{\tau})\left[E_{\tau}\right]$ and $\partial_{2} M U_{*}(\bar{\Theta})$ is represented by the pullback of the lower short exact sequence along $M U_{*}(\bar{\Theta})$.

Forming the pushout of the top sequence using $M U_{*}(\tilde{\tau})$ and the pullback of the lower sequence via $M U_{*}(\bar{\Theta})$ gives the Yoneda-equivalent short exact sequences, which therefore define the same class in $\mathrm{Ext}^{1}$, as required.

REMARK 4.10. It is instructive to check this result directly at the level of cocycles by using the description of the connecting morphism given in Lemma A.7.

4.4. Relating $K$ and $M U_{*}(\theta) \circ \sigma^{\prime}$. The morphism $M U_{*} M U \rightarrow M U_{*} K U$ associated to the orientation of $K U$ induces the morphism (using the notation introduced in Section A.2):

$$
M U_{*} K_{K U_{*}} \in \operatorname{Hom}_{M U_{*}}\left(M U_{*}\left(\mathbb{C} \mathbf{P}_{0}^{\infty} \wedge \mathbb{C P}_{0}^{\infty}\right), M U_{*} K U \otimes \mathbb{Q}[-4]\right) .
$$


This can be identified with the composite

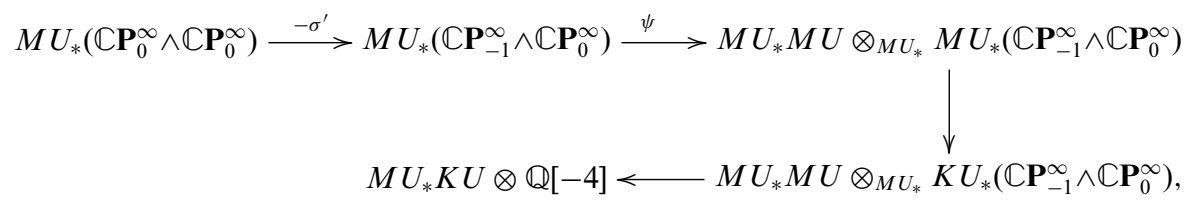

where $\psi$ is the comodule structure map, the vertical arrow is induced by the orientation of $K U$ and the final morphism of $M U_{*} M U$-modules is defined by the composite

$$
K U_{*}\left(\mathbb{C} \mathbf{P}_{-1}^{\infty} \wedge \mathbb{C} \mathbf{P}_{0}^{\infty}\right) \rightarrow K U_{*}\left(\mathbb{C} \mathbf{P}_{0}^{\infty}\right)[-2] \stackrel{K U_{*}(U) \circ \sigma}{\longrightarrow} K U_{*} \otimes \mathbb{Q}[-4]
$$

of the projection induced by $K U_{*}\left(\mathbb{C} \mathbf{P}_{-1}^{\infty}\right) \rightarrow K U_{*}[-2]$ with the morphism induced by the rational Thom class of $\mathbb{C} \mathbf{P}_{-1}^{\infty}$.

REMARK 4.11. The sign arises due to the conventions used in defining the cobar complex, as in Proposition A.8.

The second morphism in (8) is related to the morphism $\tilde{\theta}_{*}: K U_{*}\left(\mathbb{C} \mathbf{P}_{-1}^{\infty} \wedge \mathbb{C} \mathbf{P}_{0}^{\infty}\right) \rightarrow$ $K U_{*} \otimes \mathbb{Q}[-4]$ via the following commutative diagram derived from Theorem 3.16:

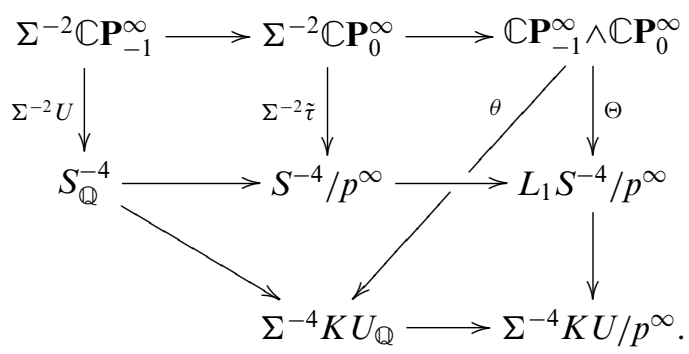

This implies the following result (which corresponds to a fundamental property of $\theta$ used in the construction of $\Theta$ ).

LEMMA 4.12. The restriction of $\tilde{\theta}_{*}: K U_{*}\left(\mathbb{C} \mathbf{P}_{-1}^{\infty} \wedge \mathbb{C} \mathbf{P}_{0}^{\infty}\right) \rightarrow K U_{*} \otimes \mathbb{Q}[-4]$ along the morphism $K U_{*}\left(\mathbb{C} \mathbf{P}_{0}^{\infty}\right)[-2] \hookrightarrow K U_{*}\left(\mathbb{C} \mathbf{P}_{-1}^{\infty} \wedge \mathbb{C P}_{0}^{\infty}\right)$, induced by the inclusion of the bottom cell $S^{-2} \hookrightarrow \mathbb{C} \mathbf{P}_{-1}^{\infty}$ is the morphism

$$
K U_{*}(U) \circ \sigma[-2]: K U_{*}\left(\mathbb{C} \mathbf{P}_{0}^{\infty}\right)[-2] \rightarrow K U_{*} \otimes \mathbb{Q}[-4] .
$$

REMARK 4.13. This result corresponds to the fact that the morphism $\tilde{\theta}_{*}$ is determined by the power series

$$
\frac{1}{S}\left(\frac{1}{\log ^{K U} T}-\frac{1}{T}\right)+\tilde{\theta}^{\prime}(S, T),
$$

where $\tilde{\theta}^{\prime}$ is the formal power series introduced in Notation 3.14.

Proposition 4.8 gives the following, using the notation of Proposition 3.17: 
Proposition 4.14. The morphism

$$
M U_{*} K_{K U_{*}} \in \operatorname{Hom}_{M U_{*}}\left(M U_{*}\left(\mathbb{C} \mathbf{P}_{0}^{\infty} \wedge \mathbb{C P}_{0}^{\infty}\right), M U_{*} K U \otimes \mathbb{Q}[-4]\right)
$$

is determined by

$$
\underline{\beta}_{0}(S) \otimes \underline{\beta}_{0}(T) \mapsto\left(\frac{1}{S}-\frac{1}{\underline{b}^{\prime}(S)}\right)\left(\frac{1}{\log ^{M U} T}-\frac{1}{\underline{b}^{\prime}(T)}\right) .
$$

Proof. By construction, $\underline{b}^{\prime}$ corresponds to the image of $\underline{b}$ under the morphism induced by $M U_{*} M U \rightarrow M U_{*} K U$, and $\log ^{L}$ maps to $\log ^{M U}$. The result follows from Proposition 4.8.

The above description of $M U_{*} K_{K U_{*}}$ can be compared with that of $M U_{*}(\theta) \circ \sigma^{\prime}$ : $M U_{*}\left(\mathbb{C} \mathbf{P}_{0}^{\infty} \wedge \mathbb{C P}_{0}^{\infty}\right) \rightarrow M U_{*} K U \otimes \mathbb{Q}[-4]$. Write $\tilde{\theta}_{*}^{\prime}$ for the morphism of $M U_{*}$-modules $M U_{*}\left(\mathbb{C} \mathbf{P}_{0}^{\infty} \wedge \mathbb{C} \mathbf{P}_{0}^{\infty}\right) \rightarrow K U_{*} \otimes \mathbb{Q}[-4]$ determined by $\tilde{\theta}^{\prime}$.

COROLlaRY 4.15. There is an identification of morphisms in $\operatorname{Hom}_{M U_{*}}\left(M U_{*}\left(\mathbb{C P}_{0}^{\infty} \wedge \mathbb{C P}_{0}^{\infty}\right), M U_{*} K U \otimes \mathbb{Q}[-4]\right):$

$$
M U_{*}(\theta) \circ \sigma^{\prime}=-M_{*} K_{K U_{*}}+\left(M U_{*} M U \otimes \tilde{\theta}_{*}^{\prime}\right) \circ \psi_{M U_{*}\left(\mathbb{C} \mathbf{P}_{0}^{\infty} \wedge \mathbb{C P}_{0}^{\infty}\right)} .
$$

Proof. Compare the calculation in Proposition 3.17 with Proposition 4.14. (Note that the sign arises from the conventions used in defining the cobar complex, as in Proposition A.8.)

5. Restricting to primitives. The spherical elements of $M U_{*}\left(\mathbb{C} \mathbf{P}_{0}^{\infty} \wedge \mathbb{C} \mathbf{P}_{0}^{\infty}\right)$ lie in the comodule primitives; this motivates the study of the restriction of the algebraic double transfer to the comodule primitives.

\subsection{Comodule primitives.}

Notation 5.1. For $M$ a left $M U_{*} M U$-comodule, write $\mathbf{P} M$ for the graded abelian group of comodule primitives.

As above, $\mathbf{H} \mathbb{Q}$ denotes the rational Eilenberg-MacLane spectrum; its integral counterpart is denoted by $\mathbf{H} \mathbb{Z}$. The following is clear:

LEMMA 5.2. For $X$ a spectrum, there is a natural commutative diagram of graded abelian groups, induced by the orientation of $\mathbf{H} \mathbb{Z}$ and rationalisation

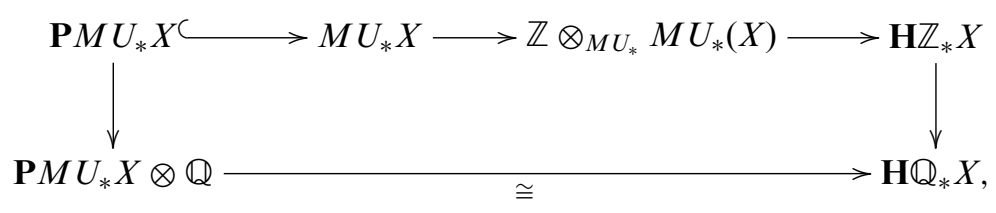

in which the lower horizontal morphism is an isomorphism.

If $M U_{*} X$ has no additive torsion, then $\mathbf{P} M U_{*} X \rightarrow \mathbf{H} \mathbb{Z}_{*} X$ is a monomorphism.

Proof. Straightforward; the isomorphism is a consequence of faithfully flat descent, since the Hopf algebroid $\left(M U_{*} \otimes \mathbb{Q}, M U_{*} M U \otimes \mathbb{Q}\right)$ is isomorphic to the unicursal 
Hopf algebroid associated to the faithfully flat morphism $\mathbb{Q} \rightarrow M U_{*} \otimes \mathbb{Q}$ (compare [17, Lemma A1.1.12]).

EXAMPLE 5.3. Let $d$ be a natural number. Then

$$
\mathbf{P} M U_{*}\left(\left(\mathbb{C} \mathbf{P}_{0}^{\infty}\right)^{\wedge d}\right) \hookrightarrow \mathbf{H} \mathbb{Z}_{*}\left(\left(\mathbb{C} \mathbf{P}_{0}^{\infty}\right)^{\wedge d}\right)
$$

is a morphism of algebras, where the product is induced by the $H$-space structure of $\mathbb{C} \mathbf{P}^{\infty}$. The homology $\mathbf{H} \mathbb{Z}_{*}\left(\left(\mathbb{C} \mathbf{P}_{0}^{\infty}\right)^{\wedge d}\right)$ is the free divided power algebra $\Gamma^{*}\left(\mathbb{Z}^{\oplus d}\right)$, hence $\mathbf{P} M U_{*}\left(\left(\mathbb{C} \mathbf{P}_{0}^{\infty}\right)^{\wedge d}\right)$ is a sub-algebra of $\Gamma^{*}\left(\mathbb{Z}^{\oplus d}\right)$.

The primitives $\mathbf{P} M U_{*}\left(\mathbb{C} \mathbf{P}_{0}^{\infty}\right)$ were calculated by Segal [18]; an elegant approach is given by Miller in [13, Proposition 4.1], where the primitive generators $p_{n} \in$ $M U_{2 n}\left(\mathbb{C} \mathbf{P}_{0}^{\infty}\right)$ are defined by means of the expansion

$$
\underline{\beta}_{0}(\exp (T))=\sum \frac{p_{n}}{n !} T^{n}
$$

in $M U_{*}\left(\mathbb{C} \mathbf{P}_{0}^{\infty}\right) \otimes \mathbb{Q}$, so that $\left|p_{n}\right|=2 n$. The morphism $\mathbf{P} M U_{*}\left(\mathbb{C} \mathbf{P}_{0}^{\infty}\right) \rightarrow \mathbf{H} \mathbb{Z}_{*}\left(\mathbb{C} \mathbf{P}_{0}^{\infty}\right)$ sends $p_{n}$ to $\left(\beta_{1}^{\mathbf{H Z}}\right)^{n}=n ! \beta_{n}^{\mathbf{H Z}}$, where the $\beta_{i}^{\mathbf{H Z}}$ denotes the canonical module generators of $\mathbf{H} \mathbb{Z}_{*}\left(\mathbb{C} \mathbf{P}_{0}^{\infty}\right)$ (cf. [13, Remark 4.2]).

By the Künneth isomorphism $M U_{*}\left(\mathbb{C} \mathbf{P}_{0}^{\infty} \wedge \mathbb{C} \mathbf{P}_{0}^{\infty}\right) \cong M U_{*}\left(\mathbb{C} \mathbf{P}_{0}^{\infty}\right) \otimes_{M U_{*}}$ $M U_{*}\left(\mathbb{C P}_{0}^{\infty}\right)$, for pairs of natural numbers $(i, j), p_{i} \otimes p_{j}$ is a primitive of $M U_{*}\left(\mathbb{C} \mathbf{P}_{0}^{\infty} \wedge \mathbb{C} \mathbf{P}_{0}^{\infty}\right)$. The integral calculation of $\mathbf{P} M U_{*}\left(\mathbb{C} \mathbf{P}_{0}^{\infty} \wedge \mathbb{C} \mathbf{P}_{0}^{\infty}\right)$ is an interesting and difficult problem: the elements $p_{i} \otimes p_{j}$ do not generate the primitives due to delicate divisibility questions (cf. $[\mathbf{1}, \mathbf{3}, \mathbf{1 0}]$, for example).

The following result follows from Lemma 5.2:

LEMMA 5.4. The primitive $\mathbf{P} M U_{*}\left(\mathbb{C} \mathbf{P}_{0}^{\infty} \wedge \mathbb{C} \mathbf{P}_{0}^{\infty}\right)$ subgroup is a graded free $\mathbb{Z}$-module such that $\mathbf{P} M U_{*}\left(\mathbb{C} \mathbf{P}_{0}^{\infty} \wedge \mathbb{C} \mathbf{P}_{0}^{\infty}\right) \otimes \mathbb{Q}$ has basis $\left\{p_{i} \otimes p_{j} \mid i, j \geq 0\right\}$.

Notation 5.5. Let $\mathfrak{p}(S, T)$ denote the two-variable power series in $M U_{*}\left(\mathbb{C} \mathbf{P}_{0}^{\infty} \wedge \mathbb{C} \mathbf{P}_{0}^{\infty}\right) \otimes \mathbb{Q}[[S, T]]:$

$$
\mathfrak{p}(S, T):=\sum_{m, n \geq 0} p_{m} \otimes p_{n} \frac{S^{m} T^{n}}{m ! n !} .
$$

LEMMA 5.6. There is an identity of formal power series:

$$
\mathfrak{p}(S, T)=\underline{\beta}_{0}(\exp S) \otimes \underline{\beta}_{0}(\exp T) .
$$

5.2. Restricting the double transfer to primitives. A primitive $\mathfrak{p}$ of degree $2 k$ in $M U_{*}\left(\mathbb{C} \mathbf{P}_{0}^{\infty} \wedge \mathbb{C} \mathbf{P}_{0}^{\infty}\right)$ corresponds to a morphism of comodules

$$
\mathfrak{p} \in \operatorname{Hom}_{M U_{*} M U}\left(M U_{*}[2 k], M U_{*}\left(\mathbb{C} \mathbf{P}_{0}^{\infty} \wedge \mathbb{C P}_{0}^{\infty}\right)\right) .
$$

This induces a class $\mathfrak{p}^{*}[\kappa] \in \operatorname{Ext}_{M U_{*} M U}^{1}\left(M U_{*}[2 k], M U_{*} \otimes \mathbb{Q} / \mathbb{Z}[-4]\right)$ and, by the chromatic connecting morphism $\partial_{1}$, the image of the double algebraic transfer:

$$
\mathfrak{p}^{*}\left[e_{\tau}^{2}\right]=\partial_{1} \mathfrak{p}^{*}[\kappa] \in \operatorname{Ext}_{M U_{*} M U}^{2}\left(M U_{*}[2 k], M U_{*}[-4]\right),
$$

where the identification follows from Proposition 4.6. 
In particular, to understand the restriction of the double algebraic transfer to the primitive element $\mathfrak{p}$, it suffices to consider $\mathfrak{p}^{*}[\kappa]$, which is represented by the cocycle $\kappa \circ \mathfrak{p}:$

$$
M U_{*}[2 k] \stackrel{\mathfrak{p}}{\longrightarrow} M U_{*}\left(\mathbb{C} \mathbf{P}_{0}^{\infty} \wedge \mathbb{C} \mathbf{P}_{0}^{\infty}\right) \stackrel{\kappa}{\longrightarrow} M U_{*} M U \otimes \mathbb{Q} / \mathbb{Z}[-4]
$$

By Proposition 4.8, the cocycle $\kappa \circ \mathfrak{p}$ fits into the commutative diagram

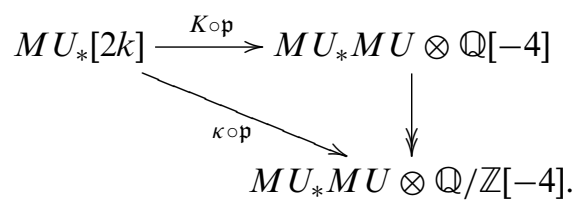

Recall that the morphism $K$ is given in Proposition 4.8 by specifying the image of $\underline{\beta}_{0}(x) \otimes \underline{\beta}_{0}(y)$.

NotATION 5.7. Write $\bar{B}_{n}^{L}, \bar{B}_{n}^{R} \in M U_{*} M U \otimes \mathbb{Q}$ for the reduced Bernoulli numbers associated to the left (respectively right) $M U_{*}$-algebra structures.

Proposition 5.8. The restriction of the morphism $K: M U_{*}\left(\mathbb{C} \mathbf{P}_{0}^{\infty} \wedge \mathbb{C P}_{0}^{\infty}\right) \rightarrow$ $M U_{*} M U \otimes \mathbb{Q}[-4]$ to the primitive elements is determined by

$$
K\left(p_{m} \otimes p_{n}\right)=\left(\bar{B}_{m+1}^{R}-\bar{B}_{m+1}^{L}\right) \bar{B}_{n+1}^{R}
$$

for natural numbers $m, n$.

Proof. By Proposition 4.8, the morphism $K$ is given by

$$
\underline{\beta}_{0}(x) \otimes \underline{\beta}_{0}(y) \mapsto\left(\frac{1}{x}-\frac{1}{\underline{b}(x)}\right)\left(\frac{1}{\log ^{L} y}-\frac{1}{\underline{b}(y)}\right) .
$$

Lemma 5.6 identifies the generating formal power series $\mathfrak{p}(S, T)$ for the primitive elements $p_{i} \otimes p_{j}$; thus, the image of $\mathfrak{p}(S, T)$ is given by substituting the power series $x=\exp ^{L} S, y=\exp ^{L} T$ in the above expression (note that the left module structure of $M U_{*} M U$ is used), which gives

$$
\mathfrak{p}(S, T) \mapsto\left(\frac{1}{\exp ^{L} S}-\frac{1}{\underline{b}\left(\exp ^{L} S\right)}\right)\left(\frac{1}{\log ^{L}\left(\exp ^{L} T\right)}-\frac{1}{\underline{b}\left(\exp ^{L} T\right)}\right) .
$$

Simplifying and reversing the order in the two brackets, this gives:

$$
\left(\frac{1}{\exp ^{R} S}-\frac{1}{\exp ^{L} S}\right)\left(\frac{1}{\exp ^{R} T}-\frac{1}{T}\right) .
$$

The result follows from the definition of $\mathfrak{p}(S, T)$ and the reduced Bernoulli numbers.

In principle, this result determines the class $\mathfrak{p}^{*}[\kappa]$, for any primitive $\mathfrak{p}$. This can be made more concrete by passing to elliptic homology and appealing to the invariants introduced by Laures [12] and Behrens [4], as explained in the following sections. 
6. Passage to elliptic homology. To study the $p$-local Adams-Novikov two-line, for $p \geq 5$ a prime, complex cobordism can usefully be replaced by elliptic homology, by change of rings. The results of this section are entirely algebraic, relying on the fact that the formal group law of elliptic homology is defined over the ring of holomorphic modular forms.

6.1. Formal group law input. Consider the ring $M F$ of holomorphic modular forms of level one over the ring $\mathbb{Z}\left[\frac{1}{6}\right]$, so that $M F \cong \mathbb{Z}\left[\frac{1}{6}\right]\left[c_{4}, c_{6}\right]$, graded by weight. The Fourier expansion at the cusp at infinity defines the $q$-expansion $M F \hookrightarrow \mathbb{Z}\left[\frac{1}{6}\right][[q]][u]$, a monomorphism of rings by the $q$-expansion principle (the variable $u$ has weight one). The morphism $q^{0}: \mathbb{Z}\left[\frac{1}{6}\right][[q]] \rightarrow \mathbb{Z}\left[\frac{1}{6}\right]$ sending a power series to its constant term induces a ring morphism $\mathbb{Z}\left[\frac{1}{6}\right][[q]][u] \rightarrow \mathbb{Z}\left[\frac{1}{6}\right][u]$. This gives a commutative diagram of ring morphisms:

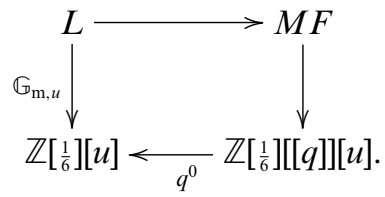

The composite $L \rightarrow \mathbb{Z}\left[\frac{1}{6}\right][[q]][u]$ classifies the (graded) formal group law associated with the Tate Weierstrass curve defined over $\mathbb{Z}\left[\frac{1}{6}\right][[q]][u]$. After reduction $q \mapsto 0$, this is the multiplicative (graded) formal group law $\mathbb{G}_{\mathrm{m}, u}$ over $\mathbb{Z}\left[\frac{1}{6}\right][[q]][u]$, since the Tate curve has multiplicative reduction.

REMARK 6.1. The universal Weierstrass curve over the ring of holomorphic modular forms $M F$ is not an elliptic curve; however, the associated formal group law is defined, since the curve is smooth at the identity section. Similarly for the Tate curve.

The ring $M F^{\text {mer }}$ of meromorphic modular forms over $\mathbb{Z}\left[\frac{1}{6}\right]$ is isomorphic to $M F\left[\Delta^{-1}\right]$, where $\Delta$ is the discriminant. The $q$-expansion of a meromorphic modular form lies in $\mathbb{Z}\left[\frac{1}{6}\right][[q]]\left[q^{-1}\right]$ and $q^{0}$ defines an additive morphism $q^{0}: \mathbb{Z}\left[\frac{1}{6}\right][[q]]\left[q^{-1}\right] \rightarrow \mathbb{Z}\left[\frac{1}{6}\right]$. This gives the following commutative diagram:

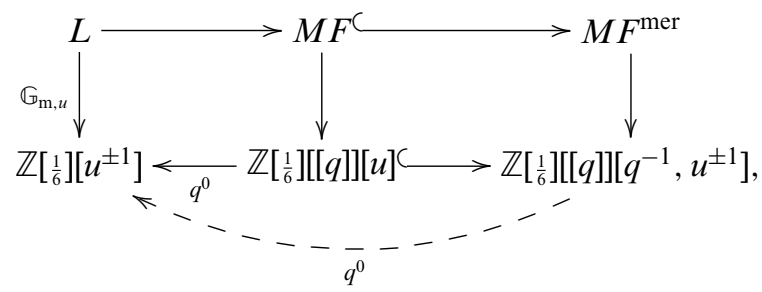

in which the solid arrows are ring morphisms.

The morphisms $L \rightarrow \mathbb{Z}\left[\frac{1}{6}\right]\left[u^{ \pm 1}\right]$ and $L \rightarrow M F^{\text {mer }}$ are Landweber exact and correspond, respectively, to $K U_{\mathbb{Z}\left[\frac{1}{6}\right]}$ (which will be denoted here simply by $K U$ ) and Ell, which denotes TMF $\left[\frac{1}{6}\right]$, the version of elliptic homology used here.

PROPOSITION 6.2. [12, Theorem 2.7]. The ring Ell ${ }_{0} \mathrm{KU}$ is isomorphic to Katz's ring $\mathfrak{D}_{\mathbb{Z}\left[\frac{1}{6}\right]}$ of divided congruences over $\mathbb{Z}\left[\frac{1}{6}\right]$. 
REMARK 6.3.

(1) The ring $E l l_{*} K U$ is concentrated in even degree and is 2-periodic.

(2) The ring $E l l_{0} K U$ is a sub-ring of $E l l_{0} K U \otimes \mathbb{Q} \cong\left(E l l_{*} \otimes K U_{*} \otimes \mathbb{Q}\right)_{0}$ and identifies with the sub- $\mathbb{Z}\left[\frac{1}{6}\right]$-module of sums $\sum_{i} f_{i}$ of modular forms such that the Fourier expansion $\Sigma_{i} f_{i}(q)$ has coefficients in $\mathbb{Z}\left[\frac{1}{6}\right]$; this is precisely the ring $\mathfrak{D}_{\mathbb{Z}\left[\frac{1}{6}\right]}$ of divided congruences [9].

6.2. The reduction map $\bar{\rho}^{1}$. The additive morphism $q^{0}: M F^{\text {mer }} \rightarrow \mathbb{Z}\left[\frac{1}{6}\right]\left[u^{ \pm 1}\right]$ is realised by a morphism of spectra $q^{0}: E l l \rightarrow K U$, which is derived from Miller's elliptic character (see [12], following Miller [14]). Hence there is an induced morphism of spectra $E l l \wedge E l l \rightarrow K U \wedge E l l$, which induces a morphism of right $E l_{*}$-modules

$$
\bar{\rho}^{1}: E l l_{*} E l l \rightarrow K U_{*} E l l,
$$

which is used in defining Laures' $f$-invariant [12] (see Section 7.1).

Since $E l l$ and $K U$ are Landweber exact, $E l l_{*} E l l \otimes \mathbb{Q} \cong E l l_{*} \otimes E l l_{*} \otimes \mathbb{Q}$ and $K U_{*} E l l \otimes \mathbb{Q} \cong K U_{*} \otimes E l l_{*} \otimes \mathbb{Q}$.

Proposition 6.4. The morphism $\bar{\rho}^{1} \otimes \mathbb{Q}$ is the morphism of right Ell $\otimes \mathbb{Q}$-modules: $q^{0} \otimes E l l_{*} \otimes \mathbb{Q}: E l l_{*} \otimes E l l_{*} \otimes \mathbb{Q} \cong E l l_{*} E l l \otimes \mathbb{Q} \rightarrow K U_{*} E l l \otimes \mathbb{Q} \cong K U_{*} \otimes E l l_{*} \otimes \mathbb{Q}$.

There is a morphism of short exact sequences of right $E l l_{*}$-modules

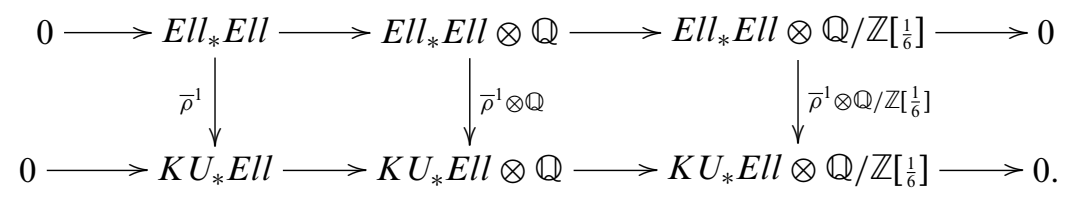

Thus, $\bar{\rho}^{1} \otimes \mathbb{Q} / \mathbb{Z}\left[\frac{1}{6}\right]$ is determined by $\bar{\rho}^{1} \otimes \mathbb{Q}$ and hence by the additive morphism $q^{0}$.

REMARK 6.5. Following [5, Theorem 4.2], the morphism $\bar{\rho}^{1}$ is used here to define the $f$-invariant rather than the analogous morphism $\rho^{1}: E l l_{*} E l l \rightarrow E l l_{*} K U$ (compare [12, Proposition 3.9]). The relationship between the two approaches to calculating the $f$-invariant is explained by [12, Proposition 3.10].

6.3. Reduction of the cocycle $\kappa$. The class $[\kappa] \in \operatorname{Ext}_{M U_{*} M U}^{1}\left(M U_{*}\left(\mathbb{C} \mathbf{P}_{0}^{\infty} \wedge\right.\right.$ $\left.\left.\mathbb{C} \mathbf{P}_{0}^{\infty}\right), M U_{*} \otimes \mathbb{Q} / \mathbb{Z}[-4]\right)$ corresponds to the algebraic double transfer, by Proposition 4.6 and, by Proposition 4.8, the representing cocycle

$$
\kappa \in \operatorname{Hom}_{M U_{*}}\left(M U_{*}\left(\mathbb{C P}_{0}^{\infty} \wedge \mathbb{C P}_{0}^{\infty}\right), M U_{*} M U \otimes \mathbb{Q} / \mathbb{Z}[-4]\right)
$$

is induced by the morphism $K \in \operatorname{Hom}_{M U_{*}}\left(M U_{*}\left(\mathbb{C P}_{0}^{\infty} \wedge \mathbb{C P}_{0}^{\infty}\right), M U_{*} M U \otimes \mathbb{Q}[-4]\right)$, by composition with the quotient map $M U_{*} M U \otimes \mathbb{Q} \rightarrow M U_{*} M U \otimes \mathbb{Q} / \mathbb{Z}$.

Base change along $M U_{*} \rightarrow E l l_{*}$ gives a cocycle

$$
\kappa_{E l l}:=E_{E l l_{*}} \kappa_{E l l_{*}} \in \operatorname{Hom}_{E l l_{*}}\left(E l l_{*}\left(\mathbb{C P}_{0}^{\infty} \wedge \mathbb{C P}_{0}^{\infty}\right), E l l_{*} E l l \otimes \mathbb{Q} / \mathbb{Z}\left[\frac{1}{6}\right][-4]\right),
$$

which represents a class $\left[\kappa_{E l l}\right] \in \operatorname{Ext}_{E l l_{*} E l l}^{1}\left(E l l_{*}\left(\mathbb{C} \mathbf{P}_{0}^{\infty} \wedge \mathbb{C P}_{0}^{\infty}\right), E l l_{*} \otimes \mathbb{Q} / \mathbb{Z}[-4]\right)$, by Lemma A.15. The following is clear: 
LEMMA 6.6. The morphism $\kappa_{\text {Ell }}$ is the reduction of the morphism

$$
E l l_{*} K_{E l l_{*}} \in \operatorname{Hom}_{E l l_{*}}\left(E l l_{*}\left(\mathbb{C P}_{0}^{\infty} \wedge \mathbb{C P}_{0}^{\infty}\right), E l l_{*} E l l \otimes \mathbb{Q}[-4]\right)
$$

via the morphism Ell ${ }_{*} E l l \otimes \mathbb{Q}[-4] \rightarrow E l l_{*} E l l \otimes \mathbb{Q} / \mathbb{Z}\left[\frac{1}{6}\right][-4]$.

Proposition 6.7. The morphism of right Ell -modules

$$
\left(\bar{\rho}^{1} \otimes \mathbb{Q} / \mathbb{Z}\left[\frac{1}{6}\right]\right) \circ \kappa_{E l l} \in \operatorname{Hom}\left(E l l_{*}\left(\mathbb{C} \mathbf{P}_{0}^{\infty} \wedge \mathbb{C P}_{0}^{\infty}\right), K U_{*} E l l \otimes \mathbb{Q} / \mathbb{Z}\left[\frac{1}{6}\right][-4]\right)
$$

coincides with the morphism $K U_{*} \kappa_{E l l_{*}}$.

Hence, the morphism $\left(\bar{\rho}^{1} \otimes \mathbb{Q} / \mathbb{Z}\left[\frac{1}{6}\right]\right) \circ \kappa_{\text {Ell }}$ is the reduction of the morphism of right Ell $_{*}$-modules

$$
K U_{*} K_{E l l_{*}} \in \operatorname{Hom}\left(E l l_{*}\left(\mathbb{C} \mathbf{P}_{0}^{\infty} \wedge \mathbb{C} \mathbf{P}_{0}^{\infty}\right), K U_{*} E l l \otimes \mathbb{Q}[-4]\right)
$$

via the morphism $K U_{*} E l l \otimes \mathbb{Q}[-4] \rightarrow K U_{*} E l l \otimes \mathbb{Q} / \mathbb{Z}\left[\frac{1}{6}\right][-4]$.

Proof. The diagram of short exact sequences (11) together with Lemma 6.6 show that it is sufficient to calculate the respective morphisms to $K U_{*} E l l \otimes \mathbb{Q}$. This can be carried out using the identification of $\bar{\rho}^{1} \otimes \mathbb{Q}$ given by Proposition 6.4.

There is a commutative diagram

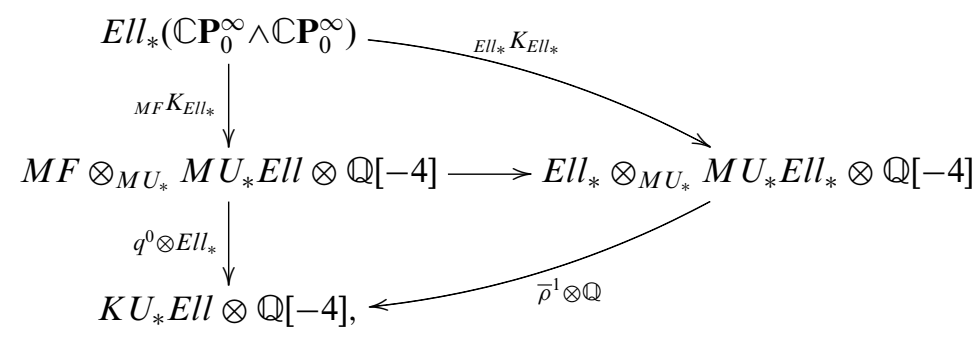

where the horizontal morphism is induced by $M F \rightarrow M F^{\text {mer }} \cong E l l_{*}$. The commutativity of the top triangle follows from the fact that the elliptic formal group law is defined over the ring of holomorphic modular forms, $M F$, and the commutativity of the lower triangle follows from the commutative Diagram (10).

To complete the proof, observe that the vertical composite is the morphism ${ }_{K U_{*}} K_{E l l_{*}}$ by the commutative Diagram (9).

7. The $f$ and $f^{\prime}$ invariants. The algebraic image of the double transfer can be analysed by using either the $f$-invariant of Laures (considered as an invariant of the Adams-Novikov two-line) or the $f^{\prime}$-invariant introduced by Behrens [4].

7.1. Recollections on the $f$-invariant. The $f$-invariant of Laures [12] is a homomorphism

$$
f: \pi_{2 k}(S) \otimes \mathbb{Z}\left[\frac{1}{6}\right] \rightarrow \mathfrak{D}_{\mathbb{Q}} /\left(\mathfrak{D}_{\mathbb{Z}\left[\frac{1}{6}\right]}+\left(M F_{0}^{\mathrm{mer}}\right)_{\mathbb{Q}}+\left(M F_{k+1}^{\mathrm{mer}}\right)_{\mathbb{Q}}\right) .
$$

This factorises across an invariant

$$
\iota^{2}: \operatorname{Ext}_{M U_{*} M U}^{2,2 k+2}\left(M U_{*}, M U_{*}\right) \otimes \mathbb{Z}\left[\frac{1}{6}\right] \hookrightarrow \mathfrak{D}_{\mathbb{Q}} /\left(\mathfrak{D}_{\mathbb{Z}\left[\frac{1}{6}\right]} \oplus\left(M F_{0}^{\mathrm{mer}}\right)_{\mathbb{Q}} \oplus\left(M F_{k+1}^{\mathrm{mer}}\right)_{\mathbb{Q}}\right),
$$

where the injectivity is given by [12, Proposition 3.9]. 
Via the chromatic connecting map

$$
\operatorname{Ext}_{M U_{*} M U}^{1, *}\left(M U_{*}, M U_{*} \otimes \mathbb{Q} / \mathbb{Z}\left[\frac{1}{6}\right]\right) \stackrel{\partial_{1}}{\rightarrow} \operatorname{Ext}_{M U_{*} M U}^{2, *}\left(M U_{*}, M U_{*}\right) \otimes \mathbb{Z}\left[\frac{1}{6}\right],
$$

$\iota^{2}$ defines an invariant of $\operatorname{Ext}_{M U_{*} M U}^{1, *}\left(M U_{*}, M U_{*} \otimes \mathbb{Q} / \mathbb{Z}\left[\frac{1}{6}\right]\right)$.

Change of rings associated to the orientation $M U_{*} \rightarrow E l l_{*}$ allows the respective groups to be replaced by

$$
\begin{aligned}
& \operatorname{Ext}_{E l l_{*} E l l}^{2, *}\left(E l l_{*}, E l l_{*}\right), \\
& \operatorname{Ext}_{E l_{*} l_{*} E l l}^{1, *}\left(E l l_{*}, E l l_{*} \otimes \mathbb{Q} / \mathbb{Z}\left[\frac{1}{6}\right]\right) .
\end{aligned}
$$

We identify the invariant $\iota^{2}$ following Behrens and Laures [5]. Write $M_{k}^{\bullet+1} \cong$ $\pi_{2 k}\left(E l l^{\bullet+1}\right)$ for the cobar complex associated to Ell. A morphism between semicosimplical abelian groups is defined [5, p. 25]:

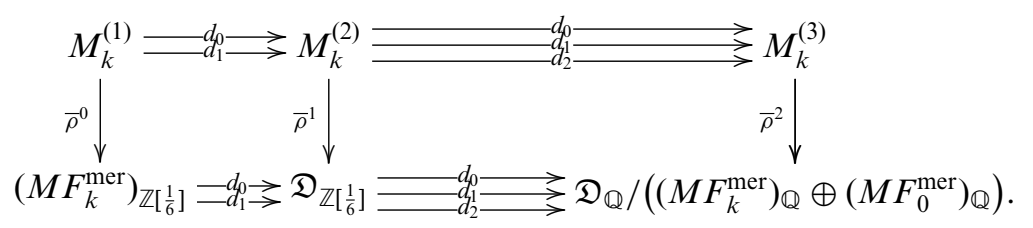

The composite of $\bar{\rho}^{2}$ with the projection

$$
\mathfrak{D}_{\mathbb{Q}} /\left(\left(M F_{k}^{\mathrm{mer}}\right)_{\mathbb{Q}} \oplus\left(M F_{0}^{\mathrm{mer}}\right)_{\mathbb{Q}}\right) \rightarrow \mathfrak{D}_{\mathbb{Q}} /\left(\mathfrak{D}_{\mathbb{Z}\left[\frac{1}{6}\right]} \oplus\left(M F_{k}^{\mathrm{mer}}\right)_{\mathbb{Q}} \oplus\left(M F_{0}^{\mathrm{mer}}\right)_{\mathbb{Q}}\right)
$$

induces the morphism

$$
\iota^{2}: \operatorname{Ext}_{E l l_{*} E l l}^{2,2 k}\left(E l l_{*}, E l l_{*}\right) \otimes \mathbb{Z}\left[\frac{1}{6}\right] \hookrightarrow \mathfrak{D}_{\mathbb{Q}} /\left(\mathfrak{D}_{\mathbb{Z}\left[\frac{1}{6}\right]} \oplus\left(M F_{0}^{\mathrm{mer}}\right)_{\mathbb{Q}} \oplus\left(M F_{k}^{\mathrm{mer}}\right)_{\mathbb{Q}}\right)
$$

on restriction to cocycles.

Write the chain co-complex associated to the cobar complex as

$$
M_{k}^{(1)} \stackrel{\delta^{0}}{\longrightarrow} M_{k}^{(2)} \stackrel{\delta^{1}}{\longrightarrow} M_{k}^{(3)} \longrightarrow \cdots
$$

The morphism $\iota^{2}$ is identified explicitly by the following straightforward application of chromatic arguments.

LEMMA 7.1. Let $x$ be a 2-cocycle in $M_{k}^{(2)}$ which represents a class

$$
[x] \in \mathrm{Ext}_{E l l_{*} E l l}^{2, *}\left(E l l_{*}, E l l_{*}\right) .
$$

Then

(1) There exists an element $c \in M_{k}^{(1)}$ and an integer $n$ such that $\delta^{1} c=n x$.

(2) The invariant $\iota^{2}[x]$ is represented by the element $\frac{1}{n} \bar{\rho}^{1}(c) \in \mathfrak{D}_{\mathbb{Q}}$.

Proof. A straightforward consequence of the commutative Diagram (12) together with the fact that $\operatorname{Ext}_{E l l_{*} E l l}^{d}\left(E l l_{*}, E l l_{*}\right) \otimes \mathbb{Q}$ is trivial for $d>0$. 
Proposition 7.2. Let $[c] \in \operatorname{Ext}_{E l l_{*} E l l}^{1,2 k}\left(E l l_{*}, E l l_{*} \otimes \mathbb{Q} / \mathbb{Z}\left[\frac{1}{6}\right]\right)$ be represented by a cocycle $c: E l l_{*}[2 k] \rightarrow$ Ell $_{*}$ Ell $\otimes \mathbb{Q} / \mathbb{Z}\left[\frac{1}{6}\right]$ which factorises as left Ell ${ }_{*}$-module morphisms

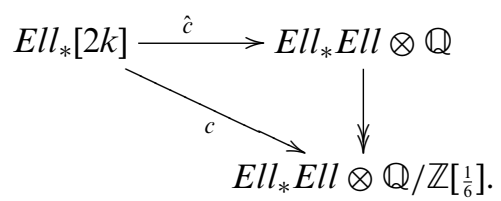

Then the invariant $\iota^{2}\left(\partial_{1}[c]\right)$ is represented by the image of the generator under the map

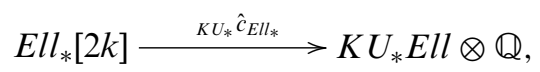

where $K U_{2 k}$ Ell $\otimes \mathbb{Q}$ is identified with $\mathfrak{D}_{\mathbb{Q}}$ by periodicity.

Proof. The result follows from Lemma 7.1.

\subsection{Restricting the $f$-invariant to primitives.}

Notation 7.3. For $\mathfrak{p}$ a primitive in $\mathbf{P} M U_{*}\left(\mathbb{C} \mathbf{P}_{0}^{\infty} \wedge \mathbb{C} \mathbf{P}_{0}^{\infty}\right)$, let $f(\mathfrak{p})$ denote the $f$ invariant of $\mathfrak{p}^{*}[\kappa] \in \operatorname{Ext}_{M U_{*} M U}^{1}\left(M U_{*}[|\mathfrak{p}|+4], M U_{*} \otimes \mathbb{Q} / \mathbb{Z}\left[\frac{1}{6}\right]\right)$.

For $n$ a natural number, write $\bar{B}_{n}^{E l l} \in E l l_{*} \otimes \mathbb{Q}$ (respectively $\bar{B}_{n}^{K U} \in K U_{*} \otimes \mathbb{Q}$ ) for the reduced Bernoulli numbers associated to the complex orientations of Ell and $K U$, respectively.

REMARK 7.4. The reduced Bernoulli number $\bar{B}_{n}^{E l l}$ is defined in the ring $M F \otimes \mathbb{Q}$ of holomorphic modular forms, since the formal group law of $E l l_{*}$ is the image of a formal group law over $M F$ via the morphism $M F \hookrightarrow M F^{\text {mer }} \cong E l l_{*}$.

THEOREM 7.5. Let $s$, $t$ be natural numbers. The $f$-invariant

$$
f\left(p_{s} \otimes p_{t}\right) \in \mathfrak{D}_{\mathbb{Q}} /\left(\mathfrak{D}_{\mathbb{Z}\left[\frac{1}{6}\right]} \oplus\left(M F_{0}^{\mathrm{mer}}\right)_{\mathbb{Q}} \oplus\left(M F_{s+t+2}^{\mathrm{mer}}\right)_{\mathbb{Q}}\right)
$$

is represented by the element $-\bar{B}_{t+1}^{E l l} \bar{B}_{s+1}^{K U} \in \mathfrak{D}_{\mathbb{Q}}$.

Proof. By Proposition 7.2, these invariants are represented by the morphism $K U_{*}(K \circ \mathfrak{p})_{E l l_{*}}$. Hence, by Proposition 5.8, $f\left(p_{s} \otimes p_{t}\right)$ is represented by

$$
\left(\bar{B}_{s+1}^{E l l}-\bar{B}_{s+1}^{K U}\right) \bar{B}_{t+1}^{E l l} \in \mathfrak{D}_{\mathbb{Q}} .
$$

The term $\bar{B}_{s+1}^{E l l} \bar{B}_{t+1}^{E l l}$ becomes zero on passage to the quotient, since it belongs to the subgroup $\left(M F_{s+t+2}^{\mathrm{mer}}\right)_{\mathbb{Q}}$.

COROLlARY 7.6. Let $s, t$ be natural numbers. The invariant $f\left(p_{s} \otimes p_{t}\right)$ is represented by the element $\bar{B}_{s+1}^{E l l} \bar{B}_{t+1}^{K U} \in \mathfrak{D}_{\mathbb{Q}}$.

Proof. The group $\mathfrak{S}_{2}$ acts on $M U_{*}\left(\mathbb{C} \mathbf{P}_{0}^{\infty} \wedge \mathbb{C} \mathbf{P}_{0}^{\infty}\right)$ by comodule morphisms induced by interchanging the factors $\mathbb{C} \mathbf{P}_{0}^{\infty}$. It is straightforward to show that the induced right action on $\operatorname{Ext}_{M U_{*} M U}^{2}\left(M U_{*}\left(\mathbb{C} \mathbf{P}_{0}^{\infty} \wedge \mathbb{C} \mathbf{P}_{0}^{\infty}\right), M U_{*}[-4]\right)$ satisfies

$$
\left(\left[e_{\tau}\right]^{2}\right) \sigma=\operatorname{sgn}(\sigma)\left[e_{\tau}\right]^{2} .
$$


Hence, $f\left(p_{s} \otimes p_{t}\right)=-f\left(p_{t} \otimes p_{s}\right)$; in particular, the $f$-invariant of $p_{s} \otimes p_{t}$ is represented by the element $\bar{B}_{s+1}^{E l l} \bar{B}_{t+1}^{K U}$.

7.3. The $f^{\prime}$-invariant on primitives. For $p \geq 5$ a prime, Behrens [4] defines the $f^{\prime}$-invariant via a morphism

$$
f^{\prime}: \operatorname{Ext}_{M U_{*} M U}^{2,2 k+2}\left(M U_{*}, M U_{*}\right)_{(p)} \rightarrow H^{0}\left(C(l)^{\bullet} / p^{\infty}, v_{1}^{\infty}\right)_{2 k+2},
$$

where $l$ is a topological generator of $\mathbb{Z}_{p}^{\times}$(for example $l=\gamma$ ) and $C(l)^{\bullet}$ is an explicit semi-cosimplicial abelian group, which is defined in terms of modular forms of level one and modular forms of level $l$. Namely, as in [4], write $M_{k}\left(\Gamma_{0}(l)\right)_{\mathbb{Z}_{p}}$ for the space of modular forms of weight $k$ and level $\Gamma_{0}(l)$ over $\mathbb{Z}_{p}$, which are meromorphic at the cusps. Then the semi-cosimplicial-graded abelian group is of the form

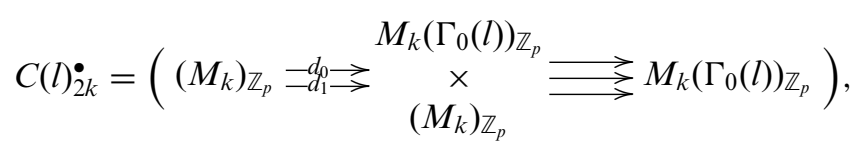

where the morphisms $d_{0}$ and $d_{1}$ are identified explicitly in terms of $q$-expansions. (See [4, Section 6] and the review in [5, Section 3].) It follows that the $f^{\prime}$-invariant of a class is represented by a modular form that satisfies certain congruences.

REMARK 7.7. Behrens and Laures [5] work $p$-locally and replace $M U$ by $B P$ so as to accord better with the results of Miller, Ravenel and Wilson [15]. Thus, below Ell $l_{*}$ denotes $p$-local elliptic homology $(p \geq 5)$, and a $p$-typical orientation $B P_{*} \rightarrow E l l_{*}$ is fixed, as in [5].

Behrens and Laures [5] show that the $f^{\prime}$-invariant fits into a commutative diagram

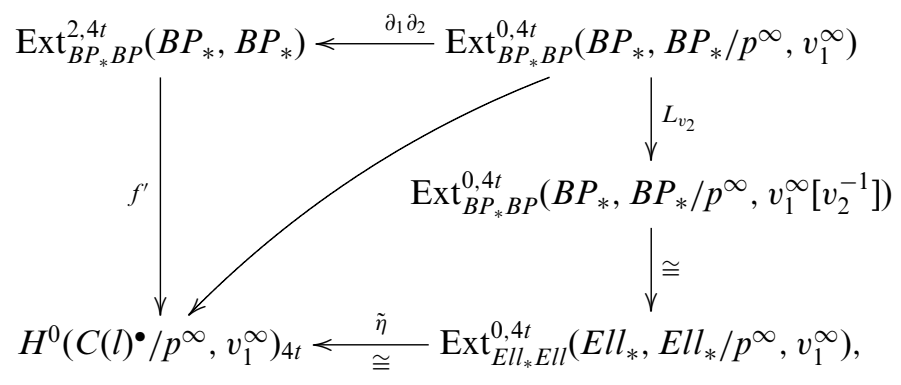

where the diagonal arrow is induced by the $p$-typical orientation of $E l l$, the vertical change of rings isomorphism is given in the proof of [5, Lemma 4.6] and the isomorphism $\tilde{\eta}$ in [5, Proposition 3.17]. The upper triangle is commutative by [5, Diagram 3.15] and the lower triangle is commutative by [5, Diagram 3.16]. Up to the isomorphism $\tilde{\eta}$, the $f^{\prime}$-invariant can be considered as taking values in the comodule primitives of $E l l_{*} / p^{\infty}, v_{1}^{\infty}$; Behrens gives a modular description of $H^{0}\left(C(l)^{\bullet} / p^{\infty}, v_{1}^{\infty}\right)_{4 t}$ in [4, Theorems 1.2 and 1.3].

Consider classes that are in the image of the algebraic double transfer. Recall that $\bar{\Theta}$ defines a chromatic factorisation of the double transfer and this induces a 
commutative diagram

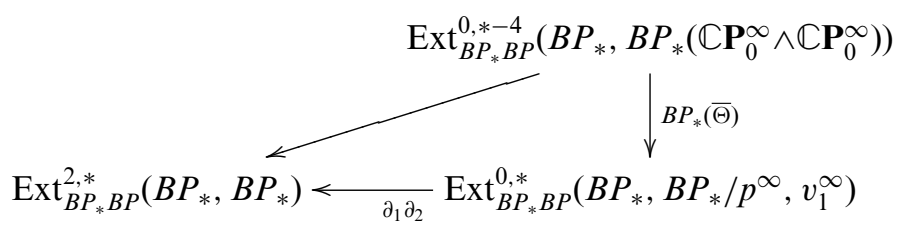

by Propositions 4.6 and 4.9 (with $B P$ in place of $M U$ ).

LEMMA 7.8. The composite morphism

$$
\begin{aligned}
\operatorname{Ext}_{B P_{*} B P}^{0,4 t-4}\left(B P_{*}, B P_{*}\left(\mathbb{C P}_{0}^{\infty} \wedge \mathbb{C P}_{0}^{\infty}\right)\right) \stackrel{B P_{*}(\bar{\Theta})}{ } & \operatorname{Ext}_{B P_{*} B P}^{0,4 t}\left(B P_{*}, B P_{*} / p^{\infty}, v_{1}^{\infty}\right) \\
& \operatorname{Ext}_{E l l_{*} E l l}^{0,4 t}\left(E l l_{*}, E l l_{*} / p^{\infty}, v_{1}^{\infty}\right)
\end{aligned}
$$

induced by the change of rings associated to the p-typical orientation $B P_{*} \rightarrow E l l_{*}$ is independent of the choice of $\bar{\Theta}$.

Proof. Follows from the commutativity of Diagrams (13) and (14), together with the fact that the bottom horizontal morphism in Diagram (13) is an isomorphism.

By naturality, it suffices to replace the composite morphism considered above by the morphism

$$
E l l_{*}(\bar{\Theta}): \operatorname{Ext}_{E l l_{*} E l l}^{0,4 t-4}\left(E l l_{*}, E l l_{*}\left(\mathbb{C} \mathbf{P}_{0}^{\infty} \wedge \mathbb{C P}_{0}^{\infty}\right)\right) \rightarrow \operatorname{Ext}_{E l l_{*} E l l}^{0,4 t}\left(E l l_{*}, E l l_{*} / p^{\infty}, v_{1}^{\infty}\right),
$$

which is considered as being the $f^{\prime}$-invariant for primitive elements. This morphism is independent of the choice of orientation; hence, in the following, $E l l_{*}$ is equipped with its standard complex orientation.

Theorem 3.16 gives a commutative diagram

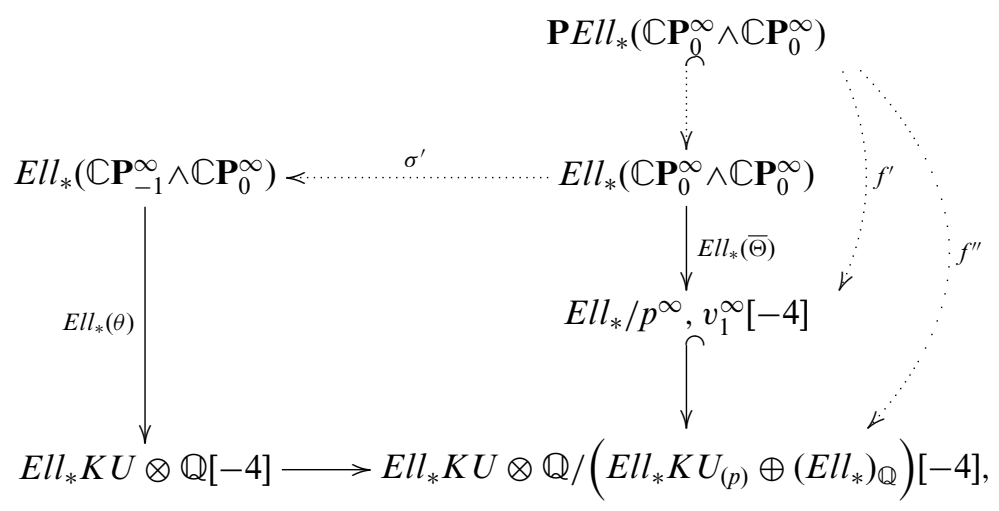

where the solid arrows denote comodule morphisms and the dotted arrows morphisms of $\mathbb{Z}_{(p)}$-modules. The composite of $f^{\prime}$ with the monomorphism $E l l_{*} / p^{\infty}, v_{1}^{\infty}[-4] \hookrightarrow$ $E l l_{*} K U \otimes \mathbb{Q} /\left(E l l_{*} K U_{(p)} \oplus\left(E l l_{*}\right)_{\mathbb{Q}}\right)[-4]$ is denoted $f^{\prime \prime}$, as indicated. 
REMARK 7.9. The morphism $E l l_{*}(\theta)$ composed with the morphism induced by $\psi^{\gamma}-1$ is integral in the appropriate sense, as a consequence of the construction of $\theta$. This can be related to the analysis of $H^{0}\left(C(l)^{\bullet} / p^{\infty}, v_{1}^{\infty}\right)$ using the identifications of [4, Proposition 6.1].

The morphism $E l l_{*}(\theta) \circ \sigma^{\prime}$ is given by Proposition 3.17, after base change to $E l l_{*}$; it is determined by $\underline{\beta}_{0}(x) \otimes \underline{\beta}_{0}(y) \mapsto$

$$
\left(\frac{1}{\underline{b}^{\prime}(x)}-\frac{1}{x}\right)\left(\frac{1}{\log ^{E l l} y}-\frac{1}{\underline{b}^{\prime}(y)}\right)+\sum_{i, j>0} \frac{B_{i}^{K U}}{i !} \frac{B_{j}^{K U}}{j !}\left(\frac{\gamma^{i}-1}{\gamma^{i+j}-1}\right)\left(\log ^{E l l} x\right)^{i-1}\left(\log ^{E l l} y\right)^{j-1},
$$

where the power series $\underline{b}^{\prime}$ is understood as $\exp ^{K U} \circ \log ^{E l l}$ when considered as a power series over $E l l_{*} K U \otimes \mathbb{Q}$.

THEOREM 7.10. The $f^{\prime}$-invariant on the primitives of $E l l_{*}\left(\mathbb{C} \mathbf{P}_{0}^{\infty} \wedge \mathbb{C} \mathbf{P}_{0}^{\infty}\right)$ is determined by

$$
f^{\prime \prime}\left(p_{s} \otimes p_{t}\right)=\left[\bar{B}_{s+1}^{E l l} \bar{B}_{t+1}^{K U}+\bar{B}_{s+1}^{K U} \bar{B}_{t+1}^{K U} \frac{\gamma^{s+1}\left(1-\gamma^{t+1}\right)}{\gamma^{s+t+2}-1}\right]
$$

where $s, t$ are natural numbers and $\bar{B}_{s+1}^{E l l} \bar{B}_{t+1}^{K U}+\bar{B}_{s+1}^{K U} \bar{B}_{t+1}^{K U} \frac{\gamma^{s+1}\left(1-\gamma^{t+1}\right)}{\gamma^{s+t+2}-1}$ is considered as an element of $E l l_{*} K U \otimes \mathbb{Q}$.

Proof. The method of proof is similar to that used in Proposition 5.8. Substitute $x=\exp ^{E l l} S$ and $y=\exp ^{E l l} T$ in the power series representing $E l l_{*}(\theta) \circ \sigma^{\prime}$; this gives the power series

$$
\left(\frac{1}{\exp ^{K U} S}-\frac{1}{\exp ^{E l l} S}\right)\left(\frac{1}{T}-\frac{1}{\exp ^{K U} T}\right)+\sum_{i, j>0} \frac{B_{i}^{K U}}{i !} \frac{B_{j}^{K U}}{j !}\left(\frac{\gamma^{i}-1}{\gamma^{i+j}-1}\right) S^{i-1} T^{j-1} .
$$

The result follows by identifying coefficients.

REMARK 7.11. The relationship between the $f$ and the $f^{\prime}$ invariants (in the general case) is made explicit in [5, Theorem 4.2] by analysing the semi-cosimplicial Diagram (12).

Upon restricting to classes arising from comodule primitives via the algebraic double transfer, the relationship is clear. Observe that Theorem 7.5, Corollary 7.6 and Theorem 7.10 show that, on passage to the quotient

$$
\mathfrak{D}_{\mathbb{Q}} /\left(\mathfrak{D}_{\mathbb{Z}\left[\frac{1}{6}\right]} \oplus\left(M F_{0}^{\mathrm{mer}}\right)_{\mathbb{Q}} \oplus\left(M F_{s+t+2}^{\mathrm{mer}}\right)_{\mathbb{Q}}\right),
$$

the elements $f\left(p_{s} \otimes p_{t}\right)$ and $f^{\prime}\left(p_{s} \otimes p_{t}\right)$ both are defined by the class of the element $\bar{B}_{s+1}^{E l l} \bar{B}_{t+1}^{K U}$ in $\mathfrak{D}_{\mathbb{Q}}$, since the additional term appearing in Theorem 7.10 becomes trivial in this quotient.

The relationship can be seen as a direct consequence of Lemma 4.12 and Corollary 4.15; here, the sign appearing in the leading term in Corollary 4.15 has been avoided by following Behrens and Lawson in defining the $f$-invariant by using the morphism $\bar{\rho}^{1}$. 
Appendix A. Cohomology and cocycles for Hopf algebroids. The reader is referred to [17, Appendix A2] for basics on Hopf algebroids. For $(A, \Gamma)$ a Hopf algebroid, the category of left $\Gamma$-comodules is denoted here $\Gamma-$ Comod and the category of $A$-modules $A$-Mod; the structure morphism of a $\Gamma$-comodule $M$ is written $\psi_{M}: M \rightarrow \Gamma \otimes_{A} M$.

A.1. Recollections on cocycles. Throughout this section, $(A, \Gamma)$ is a flat Hopf algebroid, so that $\Gamma-$ Comod is abelian with enough injectives; extension groups in this category are denoted by $\operatorname{Ext}_{\Gamma}$; the extended comodule functor $\Gamma \otimes_{A}-: A-\operatorname{Mod} \rightarrow$ $\Gamma-$ Comod is right adjoint to the forgetful functor $\Gamma-\operatorname{Comod} \rightarrow A-$ Mod.

For $M$ a $\Gamma$-comodule, let $\left(\tilde{D}_{\Gamma}^{\bullet} M, d^{\bullet}\right)$ denote the unreduced cobar resolution of $M$ (Cf. [17, Definition A1.2.11]).

Definition A.1.

(1) A $\Gamma$-comodule $X$ is $A$-projective if its underlying $A$-module is projective.

(2) A $\Gamma$-comodule $J$ is relatively injective if it has the morphism extension property with respect to monomorphisms of $\Gamma$-comodules which are split as $A$-module morphisms.

LEMMA A.2. A Г-comodule $J$ is relatively injective if and only if it is a direct summand of an extended comodule.

Lemma A.3. Let $M$ be a $\Gamma$-comodule and $N$ an A-module. Then there is a natural isomorphism

$$
\operatorname{Ext}_{\Gamma}^{i}\left(M, \Gamma \otimes_{A} N\right) \cong \operatorname{Ext}_{A}^{i}(M, N) .
$$

Hence, if $X$ is an A-projective $\Gamma$-comodule and $J$ is a relatively injective $\Gamma$-comodule,

$$
\operatorname{Ext}_{\Gamma}^{i}(X, J)= \begin{cases}\operatorname{Hom}_{\Gamma}(X, J) & i=0 \\ 0 & i>0 .\end{cases}
$$

Proof. The result follows from the argument of [17, Lemma A1.2.8b)].

Proposition A.4. [17, Lemma A.1.1.6, Corollary A1.2.12] Let $X$ be an A-projective $\Gamma$-comodule and $M$ be $a \Gamma$-comodule. Then $\operatorname{Ext}_{\Gamma}^{\circ}(X, M)$ can be calculated by a resolution of $M$ of the from $M \rightarrow J^{\bullet}$, where each $J^{k}$ is relatively injective. In particular, $\operatorname{Ext}_{\Gamma}^{\bullet}(X, M)$ is naturally isomorphic to the cohomology of the co-complex $\operatorname{Hom}_{\Gamma}\left(X, \tilde{D}_{\Gamma}^{\bullet} M\right)$, which has terms $\operatorname{Hom}_{A}\left(X, \Gamma^{\otimes_{A} S} \otimes_{A} M\right)$.

Corollary A.5. Let $X$ be an A-projective $\Gamma$-comodule and $M$ be a $\Gamma$-comodule. An extension class $[\kappa] \in \operatorname{Ext}_{\Gamma}^{1}(X, M)$ is represented by a cocycle $\kappa \in \operatorname{Hom}_{A}\left(X, \Gamma \otimes_{A} M\right)$.

The following statement makes explicit the first differential $d_{M}^{0}$ of the complex $\operatorname{Hom}_{\Gamma}\left(X, \tilde{D}_{\Gamma}^{\bullet} M\right)$.

Lemma A.6. Let $X, M$ be as in Proposition A.4 and let $\alpha: M \rightarrow N$ be a morphism of $\Gamma$-comodules.

(1) The differential $d_{M}^{0}: \operatorname{Hom}_{A}(X, M) \rightarrow \operatorname{Hom}_{A}\left(X, \Gamma \otimes_{A} M\right)$ is given by $d_{M}^{0} g:=$ $(\Gamma \otimes g) \circ \psi_{X}-\psi_{M} \circ g$.

(2) The differential $d_{N}^{0}: \operatorname{Hom}_{A}(X, N) \rightarrow \operatorname{Hom}_{A}\left(X, \Gamma \otimes_{A} N\right)$ satisfies the relation $d_{N}^{0}(\alpha \circ g)=\left(\Gamma \otimes_{A} \alpha\right) \circ d_{M}^{0} g$. 
Lemma A.7. Let $X$ be an A-projective $\Gamma$-comodule and

$$
0 \rightarrow N_{1} \rightarrow N_{2} \rightarrow N_{3} \rightarrow 0
$$

be a short exact sequence of $\Gamma$-comodules.

For a morphism of $\Gamma$-comodules $h: X \rightarrow N_{3}$ and any lift $\tilde{h}: X \rightarrow N_{2}$ of $h$ as a morphism of A-modules:

(1) The morphism $\Delta \tilde{h}:=(\Gamma \otimes \tilde{h}) \circ \psi_{X}-\psi_{N_{2}} \circ \tilde{h}$ lies in the image of $\operatorname{Hom}_{A}\left(X, \Gamma \otimes_{A}\right.$ $\left.N_{1}\right) \hookrightarrow \operatorname{Hom}_{A}\left(X, \Gamma \otimes_{A} N_{2}\right)$.

(2) The morphism $\Delta \tilde{h}: X \rightarrow \Gamma \otimes_{A} N_{1}$ is a cocycle and the associated class in $\operatorname{Ext}_{\Gamma}^{1}\left(X, N_{1}\right)$ satisfies $[\Delta \tilde{h}]=\partial h$, where $\partial: \operatorname{Hom}_{\Gamma}\left(X, N_{3}\right) \rightarrow \operatorname{Ext}_{\Gamma}^{1}\left(X, N_{1}\right)$ is the connecting morphism.

In the presence of a given module splitting of a short exact sequence of comodules, there is a useful description of a representing cocyle.

Proposition A.8. Let

$$
0 \rightarrow M \rightarrow \mathcal{E} \stackrel{p}{\rightarrow} X \rightarrow 0
$$

be a short exact sequence of $\Gamma$-comodules, in which $X$ is A-projective.

Then, for any choice of section $\sigma: X \rightarrow \mathcal{E}$, with associated retract $r: \mathcal{E} \rightarrow M$, the composite:

$$
X \stackrel{-\sigma}{\rightarrow} \mathcal{E} \stackrel{\psi_{\mathcal{E}}}{\rightarrow} \Gamma \otimes_{A} \mathcal{E} \stackrel{\Gamma \otimes r}{\rightarrow} \Gamma \otimes_{A} M
$$

is a cocycle which represents the extension class $\partial 1_{X}$, which corresponds to the short exact sequence (15). A different choice of splitting as A-modules gives rise to a cohomologous cocycle.

Proof. Consider the cocycle given by Lemma A.7, where $h$ is taken to be the identity morphism of $X$ and $\tilde{h}$ the section $\sigma$. Then the morphism $\Delta \tilde{h}$ is the difference $(\Gamma \otimes \sigma) \circ$ $\psi_{X}-\psi_{\mathcal{E}} \circ \sigma$, considered (by abuse of notation) also as a morphism to the image of $\Gamma \otimes_{A} M$ in $\Gamma \otimes_{A} \mathcal{E}$. Hence, composing with the retraction $\Gamma \otimes r$ gives $\Delta \tilde{h}=(\Gamma \otimes r) \Delta \tilde{h}$; now $r \circ \sigma=0$, so that $\Delta \tilde{h}=-(\Gamma \otimes r) \circ \psi_{\mathcal{E}} \circ \sigma$, as required. The final statement is clear.

A.2. Base change and Landweber exactness. Let $(A, \Gamma)$ be a flat Hopf algebroid and let $B \stackrel{b}{\leftarrow} A \stackrel{c}{\rightarrow} C$ be ring morphisms.

Notation A.9. Write ${ }_{B} \Gamma_{C}$ for the ring $B \otimes_{A} \Gamma \otimes_{A} C$ and $\Gamma_{B}$ (respectively $\Gamma_{C}$ ) for the ring ${ }_{B} \Gamma_{B}$ (respectively ${ }_{C} \Gamma_{C}$ ).

There are induced Hopf algebroids $\left(B, \Gamma_{B}\right)$ and $\left(C, \Gamma_{C}\right)$. The ring ${ }_{B} \Gamma_{C}$ has a left $\left(B, \Gamma_{B}\right)$ - right $\left(C, \Gamma_{C}\right)$-bicomodule structure and the structure morphisms are algebra morphisms.

Definition A.10. [6] A morphism $b: A \rightarrow B$ is Landweber exact with respect to $(A, \Gamma)$ if the functor $B \otimes_{A}-: \Gamma-$ Comod $\rightarrow B-$ Mod is exact.

Proposition A.11. [6] If $b: A \rightarrow B$ is Landweber exact with respect to $(A, \Gamma)$, then $\left(B, \Gamma_{B}\right)$ is a flat Hopf algebroid and $B \otimes_{A}-$ induces an exact functor $B \otimes_{A}-$ : $\Gamma-$ Comod $\rightarrow \Gamma_{B}-$ Comod. 
Notation A.12. For $f: X \rightarrow \Gamma \otimes_{A} M$ a morphism of left A-modules, write ${ }_{B} f_{C}$ : $B \otimes_{A} X \rightarrow{ }_{B} \Gamma_{C} \otimes_{A} M$ for the morphism of left $B$-modules:

$$
B \otimes_{A} X \stackrel{B \otimes f}{\rightarrow} B \otimes_{A} \Gamma \otimes_{A} M \stackrel{B \otimes A \otimes c \otimes M}{\rightarrow}{ }_{B} \Gamma_{C} \otimes_{A} M
$$

Example A.13. Let $X, M$ be as in Proposition A.4; a cocycle $\kappa: X \rightarrow \Gamma \otimes_{A} M$ induces a morphism ${ }_{B} \kappa_{C}: B \otimes_{A} X \rightarrow{ }_{B} \Gamma_{C} \otimes_{A} M \cong{ }_{B} \Gamma_{C} \otimes_{C}\left(C \otimes_{A} M\right)$.

Lemma A.14. Let $f: X \rightarrow \Gamma \otimes_{A} M$ and $g: M \rightarrow N$ be morphisms of left $A$ modules. There is an identity

$$
{ }_{B}\left(\left(\Gamma \otimes_{A} g\right) \circ f\right)_{C}=\left({ }_{B} \Gamma_{C} \otimes_{C}\left(C \otimes_{A} g\right)\right) \circ{ }_{B} f_{C} .
$$

Lemma A.15. Let $A \stackrel{b}{\rightarrow} B$ be a Landweber exact morphism for $(A, \Gamma)$ and $X, M$ be $\Gamma$-comodules such that $X$ is A-projective.

Let $\kappa: X \rightarrow \Gamma \otimes_{A} M$ be a cocycle representing an extension $[\kappa] \in \operatorname{Ext}_{\Gamma}^{1}(X, M)$. Then $B \otimes_{A} X$ is a B-projective $\Gamma_{B}$-comodule and the morphism ${ }_{B} \kappa_{B}: B \otimes_{A} X \rightarrow \Gamma_{B} \otimes_{B}$ $\left(B \otimes_{A} M\right)$ is a cocycle that represents the class $B \otimes_{A}[\kappa] \in \operatorname{Ext}_{\Gamma_{B}}^{1}\left(B \otimes_{A} X, B \otimes_{A} M\right)$.

\section{REFERENCES}

1. A. Baker, On the detection of some elements in the image of the double transfer using K(2)-theory, Math. Z. 197(3) (1988), 439-454. MR MR926851 (89c:55019)

2. A. Baker, D. Carlisle, B. Gray, S. Hilditch, N. Ray and R. Wood, On the iterated complex transfer, Math. Z. 199(2) (1988), 191-207. MR MR958648 (90d:55021)

3. A. Baker, F. Clarke, N. Ray and L. Schwartz, On the Kummer congruences and the stable homotopy of BU, Trans. Amer. Math. Soc. 316(2) (1989), 385-432. MR MR942424 (90c:55003)

4. M. Behrens, Congruences between modular forms given by the divided $\beta$ family in homotopy theory, Geom. Topol. 13(1) (2009), 319-357. MR MR2469520 (2009i:55016)

5. M. Behrens and G. Laures, $\beta$-family congruences and the $f$-invariant, new topological contexts for Galois theory and algebraic geometry (BIRS 2008), Geom. Topol. Monogr. 16 (2009), 9-29. (Geom. Topol. Publ., Coventry) MR MR2544384

6. M. Hovey and N. Strickland, Comodules and Landweber exact homology theories, Adv. Math. 192(2) (2005), 427-456. MR MR2128706 (2006e:55007)

7. M. Imaoka, Double transfers at the prime 2, Sūrikaisekikenkyūsho Kōkyūroku $\mathbf{8 3 8}$ (1993), 1-8. (Developments and prospects in algebraic topology (Japanese), Kyoto, Japan). MR MR1289907

8. M. Imaoka, Factorization of double transfer maps, Osaka J. Math. 30(4) (1993), 759769. MR MR1250782 (95d:55015)

9. N. M. Katz, Higher congruences between modular forms, Ann. Math. 101(2) (1975), 332-367. MR MR0417059 (54 \#5120)

10. K. Knapp, Some applications of $K$-theory to framed bordism, e-invariant and the transfer, mimeographed notes (Habilitationschrift, Universität Bonn, Germany, 1979).

11. K. Knapp, Introduction to nonconnective $\operatorname{Im}(J)$-theory, in Handbook of algebraic topology (James I. M., Editor) (North-Holland, Amsterdam, 1995), 425-461. MR MR1361896 (97a:55016)

12. G. Laures, The topological $q$-expansion principle, Topology 38(2) (1999), 387-425. MR MR1660325 (2000c:55009)

13. H. Miller, Universal Bernoulli numbers and the $S^{1}$-transfer, in Current trends in algebraic topology, Part 2 (London, Ont., 1981), CMS Conference Proceedings, vol. 2, American Mathematical Society, Providence, RI, 1982, pp. 437-449. MR MR686158 (85b:55029) 
14. H. Miller, The elliptic character and the Witten genus, Algebraic topology (Proceedings of the International Conference, Evanston, IL, 1988), Contemp. Math. 96 (1989), 281-289 (American Mathematical Society, Providence, RI, 1989). MR MR1022688 (90i:55005)

15. H. R. Miller, D. C. Ravenel and W. S. Wilson, Periodic phenomena in the AdamsNovikov spectral sequence, Ann. Math. (2) 106(3) (1977), 469-516. MR MR0458423 (56 \#16626)

16. D. C. Ravenel, Localization with respect to certain periodic homology theories, Amer. J. Math. 106(2) (1984), 351-414. MR MR737778 (85k:55009)

17. D. C. Ravenel, Complex cobordism and stable homotopy groups of spheres, in Pure and applied mathematics (Ravenel D. C., Editor), vol. 121 (Academic Press, Orlando, FL, 1986). MR MR860042 (87j:55003), $\mathrm{xx}+413$.

18. D. M. Segal, The cooperations of $M \mathrm{U}_{*}\left(\mathbf{C} P^{\infty}\right)$ and $M \mathrm{U}_{*}\left(H P^{\infty}\right)$ and the primitive generators, J. Pure Appl. Algebra 14(3) (1979), 315-322. MR MR533431 (80j:55006)

19. R. M. Switzer, Algebraic topology-homotopy and homology, in Classics in mathematics (Springer-Verlag, Berlin, Germany, 2002), Reprint of the 1975 original Springer, New York; MR0385836 (52 \#6695). MR 1886843 